TRANSACTIONS OF THE

AMERICAN MATHEMATICAL SOCIETY

Volume 349, Number 2, February 1997, Pages 809-835

S 0002-9947(97)01815-1

\title{
STIEFEL-WHITNEY CLASSES AND THE CONORMAL CYCLE OF A SINGULAR VARIETY
}

\author{
JOSEPH H. G. FU AND CLINT MCCRORY
}

\begin{abstract}
A geometric construction of Sullivan's Stiefel-Whitney homology classes of a real analytic variety $X$ is given by means of the conormal cycle of an embedding of $X$ in a smooth variety. We prove that the Stiefel-Whitney classes define additive natural transformations from certain constructible functions to homology. We also show that, for a complex analytic variety, these classes are the mod 2 reductions of the Chern-MacPherson classes.
\end{abstract}

We present a new definition of the Stiefel-Whitney homology classes of a possibly singular real analytic variety $X$. The original definition, due to Sullivan [S], involves a triangulation of $X$; its geometric meaning is unclear. Our definition uses the conormal cycle of an embedding of $X$ in a smooth variety. The conormal cycle of a subanalytic subset $X$ of an analytic manifold $M$ was defined by the first author [F4] using geometric measure theory. The conormal cycle is an integral current representing (up to sign) Kashiwara's characteristic cycle of the sheaf $D_{M} \mathbb{R}_{X}[\mathrm{~F} 4,4.7]$. Our construction of the Stiefel-Whitney classes is based on the fundamental observation that the conormal cycle of a real analytic variety is antipodally symmetric mod 2. We use this observation to give a definition of Stiefel-Whitney classes which is parallel to the first author's definition of the Chern-Schwartz-MacPherson classes of a complex analytic variety [F5]. (This definition of Chern classes is related to earlier work of Brylinski-Dubson-Kashiwara [BDK] and Sabbah [Sa].) In fact we show that the Stiefel-Whitney homology classes of a complex analytic variety are the mod 2 reductions of the Chern classes. We prove that our Stiefel-Whitney classes satisfy axioms similar to the Deligne-Grothendieck axioms for Chern classes, and we prove a specialization formula for the Stiefel-Whitney classes of a family of varieties. We show that the Stiefel-Whitney classes of an affine real analytic variety $X$ are represented by the polar cycles of $X$, introduced for simplicial spaces by Banchoff [B] and McCrory [Mc], and we give a new proof of the combinatorial formula for Stiefel-Whitney classes of manifolds.

Our central result is a specialization formula for the conormal cycle (Theorem 3.7). We use this formula to prove the basic results of Kashiwara and Schapira's calculus of subanalytically constructible functions [Sc], [KS, 9.7], as well as the pushforward and specialization formulas for Stiefel-Whitney classes.

Received by the editors October 2, 1995.

1991 Mathematics Subject Classification. Primary 14P25, 57R20; Secondary 14P15, 49Q15.

Key words and phrases. Stiefel-Whitney class, real analytic set, conormal cycle, characteristic cycle, polar cycle, integral current.

Research supported in part by NSF grant DMS-9403887. First author also partially supported by NSF grant DMS-9404366. We thank Adam Parusiński for his encouragement and help.

(C)1997 American Mathematical Society 
An advantage of using geometric measure theory to define Chern classes and Stiefel-Whitney classes is that explicit representative currents can be constructed. The geometric operations of slicing and limits of subanalytic currents correspond directly to intersection and specialization of homology classes. Integral currents have been used by Hardt and McCrory [HM] to define Steenrod operations, and by Harvey and Zweck [HZ] to define Stiefel-Whitney classes of vector bundles.

\section{Combinatorial Stiefel-Whitney Classes}

In his 1935 thesis, Stiefel defined a characteristic class $w_{i}(X)$ in the mod 2 homology of $X$, for every smooth manifold $X$ and each $i \geq 0$ : $w_{i}(X)$ is the homology class of the singular locus of a general set of $i+1$ vector fields on $X$ [St]. He conjectured that $w_{i}(X)$ is represented by the sum of all the $i$-simplices in the barycentric subdivision of a triangulation of $X$ [St, p. 342]. This conjecture was proved in 1939 by Whitney, who published a sketch of his proof [W]. Whitney also reinterpreted Stiefel's classes as characteristic cohomology classes of the tangent bundle of $X$.

Cheeger's rediscovery and proof of the combinatorial formula for Stiefel-Whitney classes of manifolds in 1969 [Ch] led to Sullivan's definition of Stiefel-Whitney homology classes of real analytic varieties with arbitrary singularities. The following result is essentially due to Sullivan $[\mathrm{S}]$; we update his result using subanalytic technology. (For a proof that every variety has a subanalytic triangulation, see [Hi2], [Ha5].)

1.1 Theorem. Let $X$ be a compact real analytic variety, let $K$ be a subanalytic triangulation of $X$, and let $K^{\prime}$ be the barycentric subdivision of $K$. For each $i \geq 0$, let $s_{i}(K) \in C_{i}\left(K^{\prime} ; \mathbb{Z} / 2 \mathbb{Z}\right)$ be the sum of all the $i$-simplices of $K^{\prime}$.

(1) The chain $s_{i}(K)$ is a cycle mod 2.

(2) The homology class $w_{i}(X)=\left[s_{i}(K)\right] \in H_{i}(X ; \mathbb{Z} / 2 \mathbb{Z})$ is independent of the triangulation $K$.

(3) If $f: X \rightarrow Y$ is a subanalytic map of real analytic varieties such that the Euler characteristic $\chi\left(f^{-1}(y)\right)$ is odd for all $y \in Y$, then $f_{*}\left(w_{i}(X)\right)=w_{i}(Y)$.

(4) If $X$ is nonsingular of dimension $d$, then $w_{i}(X)$ is Poincaré dual to the $(d-i)$ th Stiefel-Whitney cohomology class of the tangent bundle of $X$.

Furthermore, $w_{i}(X)$ is uniquely characterized by (3) and (4).

Proof. Statement (1) of the theorem follows from the fact that $X$ is a $(\bmod 2)$ Euler space [A]. Several proofs are known that real analytic varieties are Euler spaces ( $c f$. $[\mathrm{S}],[\mathrm{BV}],[\mathrm{Ha} 2])$; a new proof is given in (4.4) below. Statements (2) and (3) follow from Sullivan's mapping cylinder argument $[\mathrm{S}]$ (cf. (4.12) below) and the uniqueness of subanalytic triangulations [SY]. A complete proof of statement (4) (for smooth triangulations) was first published by Halperin and Toledo [HT]. (The method of Cairns [C], applied to a real analytic submanifold of Euclidean space, gives a triangulation which is both subanalytic and smooth.) The uniqueness of $w_{i}(X)$ follows from the representability of mod 2 homology by analytic manifolds (cf. (4.15) below): For every compact real analytic variety $X$ there exists a subanalytic map $f: V \rightarrow X$ such that $V$ is a compact smooth analytic variety of the same dimension as $X$ and $f_{*}[V]=[X]$, where $[X]$ is the mod 2 fundamental class of $X[\mathrm{BH}]$. It follows that for every compact real analytic variety $X$ there exists a subanalytic map $\varphi: W \rightarrow X$ such that $W$ is a finite disjoint union of compact 
smooth analytic varieties (of various dimensions) and $\chi\left(\varphi^{-1}(X)\right)$ is odd for all $x \in X$.

The chain $s_{i}(K)$ is the $i$ th Stiefel chain of the triangulation $K$. The homology class $w_{i}(X)$ is the $i$ th Stiefel-Whitney homology class of the variety $X$. Clearly $\epsilon w_{0}(X) \equiv \chi(X)(\bmod 2)$, where $\epsilon: H_{0}(X ; \mathbb{Z} / 2 \mathbb{Z}) \rightarrow \mathbb{Z} / 2 \mathbb{Z}$ is the augmentation homomorphism. Also, if $X$ has pure dimension $d$, then $w_{d}(X)=[X]$, the $\bmod 2$ fundamental class of $X$. However, from the combinatorial viewpoint the significance of the classes $w_{i}(X)$ for $0<i<d$ is not apparent.

\section{The Conormal Cycle}

Let $X$ be a compact subanalytic subset of the $n$-dimensional oriented real analytic manifold $M$, and let $f: M \rightarrow[0, \infty)$ be a proper, locally Lipschitz subanalytic function with $f^{-1}(0)=X$. Let $\pi: T^{*} M \rightarrow M$ be the projection, let $S^{*} M$ be the cotangent ray space of $M$, and let $\nu: T^{*} M-(0) \rightarrow S^{*} M$ be the quotient map.

2.1 Definition. The conormal cycle of $X$ in $M$ is the $(n-1)$-dimensional closed Legendrian integral current $N_{M}^{*}(X)$ in $S^{*} M$ given by

$$
N_{M}^{*}(X)=\lim _{\epsilon \rightarrow 0} \nu_{*}\langle\llbracket d f \rrbracket, f \circ \pi, \epsilon\rangle .
$$

This definition is due to $\mathrm{Fu}[\mathrm{F} 3][\mathrm{F} 4]$. Here $\llbracket d f \rrbracket$ denotes the differential current of $f[\mathrm{~F} 1]$. The expression $\langle\llbracket d f \rrbracket, f \circ \pi, \epsilon\rangle$ denotes the slice of the current $\llbracket d f \rrbracket$ by the fiber over $\epsilon$ of the function $f \circ \pi$, and the limit is taken in the flat norm topology. Since a real-valued subanalytic function has isolated critical points, the support of $\langle\llbracket d f \rrbracket, f \circ \pi, \epsilon\rangle$ is contained in $T^{*} M-(0)$ for $\epsilon$ sufficiently small. Here we use the theory of slicing for subanalytic integral currents, as developed by Hardt [Ha3].

The current $N_{M}^{*}(X)$ is independent of the choice of function $f$ defining $X$. This follows from a Morse-theoretic characterization of the local multiplicity of the conormal cycle, which we now describe. Suppose $X \subset \mathbb{R}^{n}$ is compact, and let $N^{*}(X)$ be the conormal cycle of $X$ in $\mathbb{R}^{n}$. Let $x \in X$, let $\xi: \mathbb{R}^{n} \rightarrow \mathbb{R}$ be a nonzero linear function, and let $[\xi] \in S^{*} \mathbb{R}^{n}$ be the corresponding ray. For almost all such $\xi$, the multiplicity at $(x,[\xi])$ of the cycle $N^{*}(X)$ equals the local change near $x$ of the Euler characteristic of the super-level sets of $\xi \mid X$.

More precisely, let

$$
\iota_{X}(x,[\xi])=\lim _{\epsilon \rightarrow 0} \lim _{\delta \rightarrow 0}\left(\left.\chi\left(X \cap B_{\epsilon}(x) \cap \xi^{-1}[\xi(x)-h, \infty)\right)\right|_{h=-\delta} ^{h=+\delta}\right),
$$

where $B_{\epsilon}(x)$ is the closed ball of radius $\epsilon$ centered at $x$. We identify $S^{*} \mathbb{R}^{n}$ with $\mathbb{R}^{n} \times S^{n-1}$, and we let $\kappa$ be the pullback to $S^{*} \mathbb{R}^{n}$ of the volume form on $S^{n-1}$, normalized to have total volume 1. The following theorem is due to $\mathrm{Fu}[\mathrm{F} 1,4.1]$.

2.3 Theorem. Let $X$ be a compact subanalytic subset of $\mathbb{R}^{n}$. The conormal cycle $N^{*}(X)$ is the unique compactly supported $(n-1)$-dimensional closed Legendrian integral current $T$ on $S^{*} \mathbb{R}^{n}$ such that, for all smooth functions $\varphi: S^{*} \mathbb{R}^{n} \rightarrow \mathbb{R}$,

$$
T(\varphi \cdot \kappa)=\int_{S^{n-1}} \sum_{x \in X} \iota_{X}(x, \theta) \varphi(x, \theta) d \theta .
$$

Equivalently, let $\rho: S^{*} \mathbb{R}^{n} \rightarrow S^{n-1}$ be the projection to the fiber. Then

$$
\langle T, \rho, \theta\rangle=\sum_{x \in X} \iota_{X}(x, \theta) \llbracket(x, \theta) \rrbracket .
$$


For almost all $\theta \in S^{n-1}$ the function $\iota_{X}(\cdot, \theta)$ is well-defined and takes nonzero values at only finitely many points.

Remarks. (1) It follows from [GM, 3.11] that $\iota_{X}(x,[\xi])=1-\chi\left(\ell^{+}\right)$, where $\ell^{+}$is the upper half-link of $X$ at the point $x$ with respect to the function $\xi$. We use the super-level sets $\xi^{-1}[a, \infty)$ rather than the sub-level sets $\xi^{-1}(-\infty, a]$ so that for a convex subset $X$ of $\mathbb{R}^{n}$, the support of the conormal cycle comprises the outward normals of $X$ rather than the inward normals.

(2) For the closed subanalytic subset $X$ of the oriented analytic manifold $M$, let $C C\left(\mathbb{R}_{X}\right)$ be Kashiwara's characteristic cycle of the sheaf $\mathbb{R}_{X}$ on $M$ [KS, 9.4]. Let $a: T^{*} M \rightarrow T^{*} M$ be the antipodal map (multiplication by -1 in the fiber). Now let $\vec{N}_{M}^{*}(X)$ be the conic Lagrangian cycle of $T^{*} M$ corresponding to the conormal cycle $N_{M}^{*}(X)$. Then

$$
a_{*} \vec{N}_{M}^{*}(X)=C C\left(\mathbb{R}_{X}\right) .
$$

For a proof of this result, see $[\mathrm{F} 4,4.7]$. It follows from [KS, 9.4.4] (or from (3.12) below) that

$$
(-1)^{n} \vec{N}_{M}^{*}(X)=C C\left(D_{M} \mathbb{R}_{X}\right),
$$

where $n=\operatorname{dim} M$ and $D_{M} \mathbb{R}_{X}$ is the dual of the sheaf $\mathbb{R}_{X}$.

If $X$ is a closed subanalytic subset of the analytic manifold $M$, by a stratification of $X$ we mean a Whitney stratification with subanalytic strata [Hi1] [Ha4]. If $\mathcal{S}$ is a stratification of $X$, then

$$
\operatorname{spt} N_{M}^{*}(X) \subset \bigcup_{S \in \mathcal{S}} n^{*} S,
$$

where $n^{*} S \subset S^{*} M$ is the conormal ray bundle of the stratum $S$ in $M$ [F4, 4.6.6].

It will be convenient to extend the definition of the conormal cycle to all subanalytic sets. If $X$ is a subanalytic subset of the oriented real analytic manifold $M$, then $X$ is locally closed in $M$, and the current $N_{M}^{*}(X)$ is uniquely determined by the following property. If $U$ is a subanalytic open subset of $M$ such that the closure $\bar{U}$ is compact and $X \cap \bar{U}$ is closed in $\bar{U}$, then

$$
N_{M}^{*}(X)\left\llcorner\pi^{-1} U=N_{M}^{*}(X \cap \bar{U})\left\llcorner\pi^{-1} U,\right.\right.
$$

and $N_{M}^{*}(X)\left\llcorner\pi^{-1} U=0\right.$ if $X \cap \bar{U}=\emptyset$.

The following theorem of $\mathrm{Fu}[\mathrm{F} 4,4.6]$ is of fundamental importance for applications of the conormal cycle.

2.6 Theorem. Let $X$ be a closed stratified subanalytic subset of the oriented analytic $n$-manifold $M$, with $\operatorname{dim} X<n$. Let $f: M \rightarrow \mathbb{R}$ be an analytic function such that for every stratum $S$ of $X, 0$ is a regular value of $f \mid S$. Then

$$
\begin{aligned}
N_{M}^{*} & \left(X \cap f^{-1}(-\infty, 0]\right) \\
\quad & =N_{M}^{*}\left(X \cap f^{-1}(-\infty, 0)\right)+j_{*}\left(\partial N_{M}^{*}\left(X \cap f^{-1}(-\infty, 0)\right) \times[0,1]\right),
\end{aligned}
$$

where $j: S^{*} M \times \mathbb{R} \rightarrow S^{*} M$ is the join map

$$
j(\xi, t)=\left[(1-t) \xi+t d f_{\pi(\xi)}\right] .
$$

In fact, $N_{M}^{*}\left(X \cap f^{-1}(-\infty, 0]\right)$ is the unique Legendrian cycle $T$ such that

1. $\operatorname{spt} T \subset \pi^{-1}\left(X \cap f^{-1}(-\infty, 0]\right)$,

2. $T\left\llcorner\pi^{-1}\left(f^{-1}(-\infty, 0)\right)=N_{M}^{*}\left(X \cap f^{-1}(-\infty, 0)\right)\right.$, and 
3. if $f(x)=0$ then $\left[-d f_{x}\right] \notin \operatorname{spt} T$.

Note that by replacing $f$ by $-f$ we have

$$
\begin{gathered}
N_{M}^{*}\left(X \cap f^{-1}[0, \infty)\right)=N_{M}^{*}\left(X \cap f^{-1}(0, \infty)\right)+k_{*}\left(\partial N_{M}^{*}\left(X \cap f^{-1}(0, \infty)\right) \times[0,1]\right), \\
k(\xi, t)=\left[(1-t) \xi-t d f_{\pi(\xi)}\right] .
\end{gathered}
$$

We make extensive use of the following product formula for conormal cycles. Let $\vec{N}_{M}^{*}(X)$ be the conic Lagrangian cycle of $T^{*} M$ corresponding to the conormal cycle $N_{M}^{*}(X)$. Then for closed subanalytic sets $X \subset M, X^{\prime} \subset M^{\prime}$, we have

$$
\vec{N}_{M \times M^{\prime}}^{*}\left(X \times X^{\prime}\right)=\vec{N}_{M}^{*}(X) \times \vec{N}_{M^{\prime}}^{*}\left(X^{\prime}\right)
$$

$[F 4,4.5]$. In terms of the conormal cycle in the ray space, this becomes

$$
\begin{aligned}
N_{M \times M^{\prime}}^{*}\left(X \times X^{\prime}\right) & =N_{M}^{*}(X) \circledast N_{M^{\prime}}^{*}\left(X^{\prime}\right) \\
& =j_{*}\left(N_{M}^{*}(X) \times[0,1] \times N_{M^{\prime}}^{*}\left(X^{\prime}\right)\right),
\end{aligned}
$$

where $j([\xi], t,[\eta])=[t \xi+(1-t) \eta] \in S^{*}\left(M \times M^{\prime}\right)$.

The relation of the conormal cycle to the Euler characteristic is reflected in two basic properties. First, the conormal cycle is additive [F4, 4.2]: If $X$ and $Y$ are closed subanalytic subsets of the oriented analytic manifold $M$, then

$$
N_{M}^{*}(X \cup Y)=N_{M}^{*}(X)+N_{M}^{*}(Y)-N_{M}^{*}(X \cap Y) .
$$

Second, the conormal cycle satisfies a Gauss-Bonnet property [F4, 1.5]: For $X$ a compact subset of $M$ as above, if $M$ is Riemannian, with Chern-Gauss-Bonnet form $\Omega \in \bigwedge^{n}(M)$ and transgression form $\Pi \in \bigwedge^{n-1}\left(S^{*} M\right)$, then

$$
N_{M}^{*}(X)(\Pi)+\int_{X} \Omega=\chi(X) .
$$

If $M=\mathbb{R}^{n}$, then the form $\kappa$ on $S^{*} M=\mathbb{R}^{n} \times S^{n-1}(2.3)$ is a (closed) transgression form. Thus if we choose $\theta \in S^{n-1}$ so that $\mathbb{R}^{n} \times \theta$ is transverse to a stratification of $\operatorname{spt} N_{\mathbb{R}^{n}}^{*}(X)$, then

$$
\left(N_{\mathbb{R}^{n}}^{*}(X) \cdot \llbracket \mathbb{R}^{n} \times \theta \rrbracket\right)(1)=\chi(X) .
$$

Here we use the notation $A \cdot B$ to denote the intersection of the integral currents $A$ and $B$, and the notation $\llbracket Z \rrbracket$ to denote the $k$-dimensional integral current associated to the oriented $k$-manifold $Z$.

As a consequence of (2.6) and the Gauss-Bonnet property (2.10) we have the following result on the Morse theory of the conormal cycle.

2.12 Proposition. Let $X$ be a compact subanalytic subset of the oriented analytic $n$-manifold $M$, with $\operatorname{dim} X<n$, and let $f: M \rightarrow \mathbb{R}$ be an analytic function. If $d f_{x} \neq 0$ and $\left[-d f_{x}\right] \notin \operatorname{spt} N_{M}^{*}(X)$ for all $x \in f^{-1}[a, b]$, and if

$$
N^{*}(X)\left\llcorner\pi^{-1}\left(f^{-1}(a)\right)=0,\right.
$$

then

$$
\chi\left(X \cap f^{-1}(-\infty, a]\right)=\chi\left(X \cap f^{-1}(-\infty, b]\right)
$$


Proof. The hypothesis implies that the join map $j$ of $(2.6)$ is well-defined and smooth on $\operatorname{spt} N^{*}(X) \cap \pi^{-1}\left(f^{-1}[a, b]\right)$. Let $T=j_{*}\left(N^{*}\left(X \cap f^{-1}(a, b)\right) \times[0,1]\right)$, an $n$-dimensional integral current. By $(2.6)$,

$$
\begin{aligned}
N^{*}( & \left.X \cap f^{-1}(-\infty, b]\right)-N^{*}\left(X \cap f^{-1}(-\infty, a]\right) \\
= & N^{*}\left(X \cap f^{-1}(a, b)\right) \\
& \quad+j_{*}\left(\partial\left(N^{*}\left(X \cap f^{-1}(-\infty, b)\right)-N^{*}\left(X \cap f^{-1}(-\infty, a)\right)\right) \times[0,1]\right) \\
= & \partial T
\end{aligned}
$$

since, by (1), $\partial\left(N^{*}(X)\left\llcorner\pi^{-1}\left(f^{-1}(-\infty, a)\right)\right)=-\partial\left(N^{*}(X)\left\llcorner\pi^{-1}\left(f^{-1}(a, \infty)\right)\right)\right.\right.$. Therefore,

$$
\begin{aligned}
\chi(X \cap & \left.f^{-1}(-\infty, b]\right)-\chi\left(X \cap f^{-1}(-\infty, a]\right) \\
& =\left(N^{*}\left(X \cap f^{-1}(-\infty, b]\right)-N^{*}\left(X \cap f^{-1}(-\infty, a]\right)\right)(\Pi) \quad \text { by }(2.10) \\
& =\partial T(\Pi) \\
& =T(d \Pi) \\
& =T\left(\pi^{*}(\Omega)\right) \\
& =\pi_{*} T(\Omega) \\
& =0,
\end{aligned}
$$

since $\operatorname{dim} \operatorname{spt} \pi_{*} T<n$.

2.13 The mod 2 conormal cycle. Let $X$ be a compact subanalytic subset of the real analytic $n$-manifold $M$. If we do not assume that $M$ is oriented, the conormal cycle of $X$ in $M$ can be defined as an integral current modulo 2 . It is the $(n-1)$-dimensional closed Legendrian integral current mod 2 given by

$$
\mathcal{N}_{M}^{*}(X)=\lim _{t \rightarrow 0} \nu_{*}\langle\llbracket f \rrbracket, f \circ \pi, \epsilon\rangle
$$

as in (2.1), where $\llbracket f \rrbracket$ is the mod 2 differential current of a non-negative function $f$ which defines $X$, and the slice and limit are in the sense of subanalytic integral currents mod 2 (cf. [Ha1], [Ha3]).

Almost all the results of this section are true for the mod 2 conormal cycle; only the Gauss-Bonnet property (2.10) requires that the ambient manifold $M$ is oriented. The proofs of the mod 2 results are parallel to the proofs in the oriented case. The main technical point is the mod 2 version of Fu's uniqueness theorem for Lagrangian cycles $[\mathrm{F} 1,1.1]$, which is used to characterize the mod 2 differential current $\llbracket f \rrbracket$ and to prove the uniqueness part of (2.6).

An alternate approach is to define the mod 2 conormal cycle in terms of local orientations of $M$. If $\left\{U_{i}\right\}$ is a covering of $M$ by oriented open sets, then $\mathcal{N}_{M}^{*}(X)$ is uniquely characterized by the property that

$$
\mathcal{N}_{M}^{*}(X)\left\llcorner\pi^{-1} U_{i}=\left\{N_{U_{i}}^{*}\left(X \cap U_{i}\right)\right\}_{2}\right.
$$

for all $i$, where $\{C\}_{2}$ denotes the mod 2 reduction of the integral current $C$. Thus the local properties of $\mathcal{N}^{*}$ can be proved by reduction mod 2 of the corresponding properties of $N^{*}$. 


\section{SPecialization}

As Parusiński has emphasized, the process of specialization is of basic importance in subanalytic homology theory. In this section we prove a specialization formula for the conormal cycle, and we apply it to derive those aspects of Kashiwara and Schapira's calculus of subanalytically constructible functions [Sc] [KS, 9.7] which are needed to state and prove the Deligne-Grothendieck axioms for Stiefel-Whitney classes. (In contrast, Kashiwara and Schapira define operations on constructible functions using standard operations of the derived category of sheaves.) We define the operations of Euler characteristic (integral), pushforward (direct image) and duality, and we prove the key relations between these operations.

3.1 Definition. Let $M$ be a real analytic manifold. The function $\alpha: M \rightarrow \mathbb{Z}$ is (subanalytically) constructible if $\alpha^{-1}(n)$ is a subanalytic set for all $n \in \mathbb{Z}$, and the collection $\left\{\alpha^{-1}(n)\right\}_{n \in \mathbb{Z}}$ is locally finite.

The set of constructible functions on $M$ is a ring, with $(\alpha+\beta)(p)=\alpha(p)+\beta(p)$ and $(\alpha \cdot \beta)(p)=\alpha(p) \beta(p)$. Every constructible function on $M$ can be written as a locally finite sum

$$
\alpha=\sum_{i} n_{i} 1_{X_{i}}
$$

where $n_{i} \in \mathbb{Z}$, each $X_{i}$ is a closed subanalytic subset of $M$, and $1_{X}$ denotes the characteristic function of $X$.

3.3 Definition. If $\alpha$ is a constructible function on $M$ with compact support, the Euler characteristic $\chi(\alpha) \in \mathbb{Z}$ is defined as follows. Write $\alpha$ as a finite sum (3.2) with each $X_{i}$ compact. Then

$$
\chi(\alpha)=\sum_{i} n_{i} \chi\left(X_{i}\right)
$$

Kashiwara and Schapira use the suggestive notation $\chi(\alpha)=\int_{M} \alpha$. By the triangulation theorem for subanalytic sets [Hi2] [Ha5], the Euler characteristic is uniquely determined by two properties:

1. $\chi(\alpha+\beta)=\chi(\alpha)+\chi(\beta)$ for all $\alpha, \beta$.

2. $\chi\left(1_{X}\right)=1$ if $X$ is subanalytically homeomorphic to a closed ball.

3.4 Definition. If $\alpha$ is a constructible function on the oriented analytic manifold $M$, the conormal cycle $N_{M}^{*}(\alpha)$ is defined as follows. Write $\alpha$ as a locally finite sum (3.2), and let

$$
N_{M}^{*}(\alpha)=\sum_{i} n_{i} N_{M}^{*}\left(X_{i}\right)
$$

a closed Legendrian integral current in the cotangent ray space $S^{*} M$.

By (2.9) the conormal cycle $N_{M}^{*}(\alpha)$ is well-defined. The Gauss-Bonnet theorem (2.10) yields

$$
N_{M}^{*}(\alpha)(\Pi)=\chi(\alpha)
$$

for a compactly supported constructible function $\alpha$ with $\operatorname{dim} \operatorname{spt} \alpha<\operatorname{dim} M$, where $\Pi$ is a transgression form for $M$. 
3.6 Proposition. If $M$ is an oriented analytic manifold, then $N_{M}^{*}$ is an isomorphism from the group of constructible functions $\alpha$ on $M$ such that $\operatorname{spt} \alpha$ has codimension at least 2 to the group of subanalytic Legendrian cycles $Z$ such that $\pi(\operatorname{spt} Z)$ has codimension at least 2.

Proof. Suppose $\alpha, \beta$ are constructible functions with support of codimension at least 2 , with $N_{M}^{*}(\alpha)=N_{M}^{*}(\beta)$ and $\operatorname{dim}(\operatorname{spt} \alpha) \geq \operatorname{dim}(\operatorname{spt} \beta)$. Let $\mathcal{S}$ be a subanalytic Whitney stratification of $M$ so that both $\alpha$ and $\beta$ are constant on the strata of $\mathcal{S}$. Let $S$ be a $k$-dimensional stratum of $\mathcal{S}$, where $k=\operatorname{dim}(\operatorname{spt} \alpha)$. Then $N_{M}^{*}(\alpha)\left\llcorner\pi^{-1}(S)=m N_{M}^{*}(S)\right.$ and $N_{M}^{*}(\beta)\left\llcorner\pi^{-1}(S)=n N_{M}^{*}(S)\right.$ for some $m, n \in \mathbb{Z}$. Thus $m=n$, and we obtain $\alpha=\beta$ by induction on $\operatorname{dim}(\operatorname{spt} \alpha)$.

On the other hand, suppose $Z$ is a subanalytic Legendrian cycle in $S^{*} M$, put $A=$ spt $Z$, and suppose that the codimension $k$ of $\pi(A)$ is at least 2 . Let $\mathcal{S}$ be a stratification of $A$ such that the restriction of $\pi$ to each $S \in \mathcal{S}$ is a submersion. The Legendre condition implies that each $S \subset \nu^{*} \pi(S)$. Let $\mathcal{V}=\left\{\pi(S)-\bigcup_{S^{\prime} \in \mathcal{S}, S^{\prime} \neq S} \operatorname{clos} \pi\left(S^{\prime}\right) \mid\right.$ $\pi(S)$ has maximal dimension $m-k\}$, where $m=\operatorname{dim} M$; this is a collection of nonempty open subanalytic submanifolds $V$ of $M$, and each $V \in \mathcal{V}$ has a neighborhood $U \subset M$ such that $\operatorname{spt}\left(Z\left\llcorner\pi^{-1}(U)\right) \subset \nu^{*} V\right.$. Since $k \geq 2$, each $\nu^{*} V$ is connected, so the constancy theorem implies that $Z\left\llcorner\pi^{-1}(U)=n_{V} \llbracket \nu^{*} V \rrbracket\right.$ for some $n_{V} \in \mathbb{Z}$. Then $\pi\left(\operatorname{spt}\left(Z-\sum n_{V} N_{M}^{*}(\bar{V})\right)\right)$ has codimension larger than $k$, and the assertion follows by induction.

The central result of this section is the following specialization formula for the conormal cycle. The following notation will be useful. If $g: M \rightarrow \mathbb{R}, X \subset M$, and $s, t \in \mathbb{R}, s<t$, let $X_{t}=X \cap g^{-1}(t)$ and $X_{[s, t]}=X \cap g^{-1}([s, t])$. For $p, q \in M$, let $\rho_{p}(q)$ denote the distance from $p$ to $q$ in an analytic metric on $M$.

3.7 Theorem. Let $\alpha$ be a constructible function on the oriented analytic manifold $M$ such that $X=\operatorname{spt} \alpha$ is compact and has positive codimension. Let $g: M \rightarrow \mathbb{R}$ be a subanalytic function. For $t \in \mathbb{R}$, put $\alpha_{t}=\alpha \cdot 1_{X_{t}}$. For each $p \in X_{0}$, there is $\epsilon_{0}>0$ such that if $0<\epsilon<\epsilon_{0}$ then the limit

$$
\phi_{0} \alpha(p)=\lim _{t \downarrow 0} \chi\left(\alpha_{t} \cdot 1_{B_{\epsilon}(p)}\right)
$$

exists, and this limit is independent of $\epsilon$. The resulting function $\phi_{0} \alpha$ is constructible, and

$$
N_{M}^{*}\left(\phi_{0} \alpha\right)=\lim _{t \downarrow 0} N_{M}^{*}\left(\alpha_{t}\right),
$$

where the limit is in the flat norm topology. More precisely, for all sufficiently small $h>0$ there is an integral current $Z_{h}$ in $S^{*} M$ such that

1. $\partial Z_{h}=N_{M}^{*}\left(\alpha_{h}\right)-N_{M}^{*}\left(\phi_{0} \alpha\right)$,

2. $\operatorname{spt} Z_{h} \subset \pi^{-1}\left(X_{[0, h]}\right)$,

3. $Z_{h} \rightarrow 0$ in mass as $h \rightarrow 0$.

Proof. For simplicity we let $N^{*}(\alpha)=N_{M}^{*}(\alpha)$. Let $\mathcal{S}$ be a Whitney stratification of $X$ such that $\alpha \mid S$ is constant and $g \mid S$ is smooth for all $S \in \mathcal{S}$. Let $h_{0}>0$ be so small that for all $S \in \mathcal{S}$ the restriction $g \mid S$ has no critical values in $\left(0, h_{0}\right]$. Put

$$
Z=\left(j_{*}+k_{*}\right)\left(\left(N^{*}(\alpha)\left\llcorner(g \circ \pi)^{-1}\left(0, h_{0}\right]\right) \times[0,1]\right),\right.
$$


where $j, k: S^{*} M \times \mathbb{R} \rightarrow S^{*} M$ are the join maps defined in (2.6). Then $Z$ is a subanalytic integral current of dimension $n=\operatorname{dim} M$, with

$$
\begin{aligned}
\langle Z, g \circ \pi, t\rangle & =\left(j_{*}+k_{*}\right)\left(\left\langle N^{*}(\alpha), g \circ \pi, t\right\rangle \times[0,1]\right) \\
& =N^{*}\left(\alpha_{t}\right)
\end{aligned}
$$

for $t \in\left(0, h_{0}\right]$, by $(2.6)$ and the addition formula (2.9). In particular,

$$
\lim _{t \downarrow 0} N^{*}\left(\alpha_{t}\right)=N^{*}\left(\alpha_{h_{0}}\right)-\partial Z
$$

exists, and by (3.6) equals $N^{*}(\psi)$ for some constructible function $\psi$. To complete the proof we must show that $\psi=\phi_{0} \alpha$; then the currents $Z_{h}=Z\left\llcorner\pi^{-1} g^{-1}(0, h]\right.$, $0<h \leq h_{0}$, satisfy the stated relations.

Let $\mathcal{T}$ be a stratification of $X$ subordinate to $\mathcal{S} \cup\left\{X_{0}\right\}$, such that $\mathcal{T}$ satisfies the $\left(a_{g}\right)$ condition $(c f .[\mathrm{Be}$, Prop. 10$])$. Then

$$
\begin{aligned}
\operatorname{spt} N^{*}(\psi) & \subset \limsup _{h \downarrow 0}\left(\operatorname{spt} N^{*}\left(\alpha_{h}\right)\right) \\
& \subset \limsup _{h \downarrow 0} \bigcup_{S \in \mathcal{S}} n^{*}\left(S_{h}\right) \\
& \subset \limsup _{h \downarrow 0} \bigcup_{T \in \mathcal{T}} n^{*}\left(T_{h}\right) \\
& \subset \bigcup_{T \in \mathcal{T}, T \subset X_{0}} n^{*} T,
\end{aligned}
$$

where the last inclusion follows from the $\left(a_{g}\right)$ condition. Now write $\psi=\sum n_{i} 1_{Y_{i}}$, $n_{i} \neq 0$, where the sum is locally finite, and $Y_{i}$ are compact and subanalytic, with $Y_{i}=\overline{Y_{i}^{\circ}}, Y_{i}^{\circ}$ smooth and $\bigcup_{i} n^{*}\left(Y_{i}^{\circ}\right) \supset \operatorname{spt} N^{*}(\psi)$, let $p \in X_{0}$ be fixed, and choose $\epsilon_{0}>0$ so small that the following two conditions hold:

1. For all $r \in\left(0, \epsilon_{0}\right), Y_{i} \cap B_{r}(p)$ is homeomorphic to a cone. (Such $\epsilon_{0}$ exists by [Ha6].)

2. There exists $c>0$ with $\left\|d_{x}\left(\rho_{p} \mid T\right)\right\| \geq c$ for all strata $T \in \mathcal{T}, T \subset X_{0}$, and all points $x \in T \cap B_{\epsilon_{0}}(p), x \neq p$.

Then, for $r<\epsilon_{0}$,

$$
\psi(p)=\sum n_{i} 1_{Y_{i}}(p)=\sum n_{i} \chi\left(Y_{i} \cap B_{r}(p)\right),
$$

and (1) implies that

$$
\left\|d_{x}\left(\rho_{p} \mid T_{h}\right)\right\| \geq c / 2
$$

whenever $h>0$ is sufficiently small, $T \in \mathcal{T}$, and $x \in T_{h} \cap B_{r}(p)$.

We claim that, if $r<\epsilon_{0}$, then

$$
\lim _{h \downarrow 0} N^{*}\left(\alpha_{h} \cdot 1_{B_{r}(p)}\right)=N^{*}\left(\psi \cdot 1_{B_{r}(p)}\right) .
$$

For the restrictions of the two sides to the interior of $B_{r}(p)$ are equal by construction. On the other hand, (1), (3) and the join construction of (2.6) imply that

$$
\operatorname{clos}\left(\bigcup_{h \leq h_{0}} \operatorname{spt} N^{*}\left(\alpha_{h} \cdot 1_{B_{r}(p)}\right)\right) \cap \operatorname{graph}\left(-d \rho_{p}\right) \backslash \pi^{-1}(p)=\emptyset,
$$


and therefore

$$
\operatorname{spt}\left(\lim _{h \downarrow 0} N^{*}\left(\alpha_{h} \cdot 1_{B_{r}(p)}\right)\right) \cap \operatorname{graph}\left(-d \rho_{p}\right) \cap \pi^{-1} S_{r}(p)=\emptyset .
$$

Now (4) follows from the uniqueness part of (2.6).

Therefore, if $\Pi$ is a transgression form for $M$, we have by (2) and (2.10) that

$$
\begin{aligned}
\phi_{0} \alpha(p) & =\lim _{h \downarrow 0} \chi\left(\alpha_{h} \cdot 1_{B_{r}(p)}\right) \\
& =\lim _{h \downarrow 0} N^{*}\left(\alpha_{h} \cdot 1_{B_{r}(p)}\right)(\Pi) \\
& =N^{*}\left(\psi \cdot 1_{B_{r}(p)}\right)(\Pi) \\
& =\psi(p),
\end{aligned}
$$

as desired.

3.8 Definition. Let $M$ and $N$ be analytic manifolds, and let $f: X \rightarrow Y$ be a subanalytic map, where $X \subset M$ and $Y \subset N$. Let $\alpha$ be a constructible function on $M$ such that $\operatorname{spt} \alpha \subset X$, and suppose that $f \mid \operatorname{spt} \alpha$ is proper. The pushforward $f_{*} \alpha$ is the constructible function on $N$, with $\operatorname{spt} f_{*} \alpha \subset Y$, given by

$$
\left(f_{*} \alpha\right)(q)=\chi\left(\alpha \cdot 1_{f^{-1}(q)}\right) .
$$

The properties of pushforward may be derived from the specialization formula, applied to the mapping cylinder. Given subanalytic subsets $X \subset \mathbb{R}^{m}, Y \subset \mathbb{R}^{n}$ and a subanalytic map $f: X \rightarrow Y$, consider the mapping cylinder of $f$,

$$
C=\{((1-t) x, t, f(x)) \mid x \in X, 0 \leq t \leq 1\} \cup\{(0,1, y) \mid y \in Y\} .
$$

This is a subanalytic subset of $\mathbb{R}^{m} \times \mathbb{R} \times \mathbb{R}^{n}$ of positive codimension. Let $g: \mathbb{R}^{m} \times$ $\mathbb{R} \times \mathbb{R}^{n} \rightarrow \mathbb{R}$ be the projection to the middle factor, and let $C_{t}=C \cap g^{-1}(t)$. Suppose now that $\alpha$ is a constructible function on $\mathbb{R}^{m}$ with compact support contained in $X$. We let $\tilde{\alpha}$ be the constructible function on $\mathbb{R}^{m} \times \mathbb{R} \times \mathbb{R}^{n}$ given by $\tilde{\alpha}((1-t) x, t, f(x))=$ $\alpha(x)$ for $x \in X, t \in[0,1)$, and $\tilde{\alpha}$ equal to zero elsewhere. For $t \in[0,1)$, let $\tilde{\alpha}_{t}=\tilde{\alpha} \cdot 1_{C_{t}}$. For $s \in[0,1]$, we define the right and left specializations of $\tilde{\alpha}_{t}$ as $t \rightarrow s$ to be the constructible functions $\phi_{s}^{ \pm} \tilde{\alpha}$ given by $\phi_{s}^{ \pm} \tilde{\alpha}(p)=\lim _{t \rightarrow s \pm} \chi\left(\tilde{\alpha}_{t} \cdot 1_{B_{\epsilon}(p)}\right)$ for sufficiently small $\epsilon>0$.

3.9 Proposition. Let $X \subset \mathbb{R}^{m}, Y \subset \mathbb{R}^{n}$ be subanalytic sets. Let $f: X \rightarrow Y$ be a subanalytic map with mapping cylinder $C$, and let $\alpha$ be a constructible function on $\mathbb{R}^{m}$ with compact support contained in $X$. Then

$$
\begin{aligned}
& \phi_{0}^{+} \tilde{\alpha}=\alpha, \\
& \phi_{1}^{-} \tilde{\alpha}=f_{*} \alpha .
\end{aligned}
$$

Furthermore, there is a subanalytic integral current $W$ with $\operatorname{spt} W \subset \pi^{-1} C$ and

$$
\partial W=N_{M}^{*}\left(f_{*} \alpha\right)-N_{M}^{*}(\alpha) .
$$

Here we identify the constructible functions $\alpha$ and $f_{*} \alpha$ with the corresponding functions on $\mathbb{R}^{m} \times\{0\} \times\{0\}$ and $\{0\} \times\{0\} \times \mathbb{R}^{n}$ respectively.

Proof. First we establish the specialization formulas.

We prove (2) first. Let $\mathcal{S}$ be a Whitney stratification of $C$ and $\mathcal{T}$ an $\left(a_{g}\right)$ stratification refining $\mathcal{S} \cup C_{1}$, as in the proof of (3.7); let $q \in C_{1}=Y$ be a given 
point. Let $\epsilon_{0}>0$ be so small that $\left\|d_{x}\left(\rho_{q} \mid T\right)\right\| \geq c_{0}>0$ for $x \in T \cap B_{\epsilon_{0}}(q), x \neq q$, $C_{1} \supset T \in \mathcal{T}$. Theorem 2.6 and the analogue of the relation (3.7)(1) imply that

$$
\left[-d_{x} \rho_{q}\right] \notin \limsup _{t \uparrow 1}\left(\operatorname{spt} N^{*}\left(\tilde{\alpha}_{t} \cdot 1_{B_{\epsilon_{0}}(q)}\right)\right)
$$

for $x \in S_{\epsilon_{0}}(q)$. Consider the modified distance function $\tilde{\rho}(x, t, y)=\rho_{q}(y)$; clearly $\rho_{q}=\tilde{\rho}$ on $C_{1}$. Thus $(4)$ and the $\left(a_{g}\right)$ condition imply that, given $\epsilon_{1} \in\left(0, \epsilon_{0}\right)$, we have for all $t$ sufficiently close to 1 ,

$$
\left[-d_{x} \tilde{\rho}\right] \notin \operatorname{spt} N^{*}\left(\tilde{\alpha}_{t} \cdot 1_{B_{\epsilon_{0}}(q)}\right)
$$

for $x \in \tilde{\rho}^{-1}\left[\epsilon_{1}, \epsilon_{0}\right]$, and, if $\epsilon_{1}$ is sufficiently small, $C_{t} \cap \tilde{\rho}^{-1}\left[0, \epsilon_{0}\right] \supset C_{t} \cap B_{\epsilon_{0}}(q) \supset$ $C_{t} \cap \tilde{\rho}^{-1}\left[0, \epsilon_{1}\right]$. Therefore for such $t(2.12)$ yields

$$
\begin{aligned}
\chi\left(\tilde{\alpha}_{t} \cdot 1_{B_{\epsilon_{0}}(q)}\right) & =\chi\left(\tilde{\alpha}_{t} \cdot 1_{\tilde{\rho}^{-1}\left[0, \epsilon_{1}\right]}\right) \\
& =\chi\left(\alpha \cdot 1_{f^{-1}\left(B_{\epsilon_{1}}(q)\right)}\right) \\
& =\chi\left(\alpha \cdot 1_{f^{-1}(q)}\right),
\end{aligned}
$$

since $C_{t} \cap \tilde{\rho}^{-1}\left[0, \epsilon_{1}\right] \cong f^{-1}\left(B_{\epsilon_{1}}(q)\right)$. The relation (2) now follows from the specialization formula (3.7).

To prove (1) we simply repeat the argument of the last paragraph with $\tilde{\rho}$ replaced by the function $\hat{\rho}(x, t, y)=\rho_{p}(x)$, where $p \in X$, and we observe that $C_{t} \cap \hat{\rho}^{-1}\left[0, \epsilon_{1}\right] \cong$ $X \cap B_{\epsilon_{1}}(p)$ for $t$ close to 0 .

Now by [Hi1, Lemma 4.8.3] and [Be, Lemma 15], given a subanalytic Whitney stratification $\mathcal{X}$ of $X$, there exists a subanalytic Whitney stratification $\mathcal{T}$ of the mapping cylinder $C$ satisfying $\left(a_{g}\right)$ and with the property that $\mathcal{T} \mid C_{[0,1)}=\mathcal{X} \times[0,1)$. More precisely, $C_{[0,1)}=\{(x, t, y) \in C \mid 0 \leq t<1\}$ is a union of strata of $\mathcal{T}$, and all the strata of $C_{[0,1)}$ are of the form $H(S \times\{0\})$ or $H(S \times(0,1))$ for $S \in \mathcal{X}$ and $H: X \times[0,1) \rightarrow C_{[0,1)}$ the homeomorphism $H(x, t)=(x, t, f(x))$. Thus for all $T \in \mathcal{T}$, the restriction $g \mid T$ has no critical values in $(0,1)$.

Therefore, by the proof of (3.7), there exist integral currents $Z^{+}, Z^{-}$in $S^{*}\left(\mathbb{R}^{m} \times \mathbb{R} \times \mathbb{R}^{n}\right)$ such that

$$
\begin{array}{ll}
\partial Z_{0}^{+}=N^{*}\left(\tilde{\alpha}_{\frac{1}{2}}\right)-N^{*}\left(\phi_{0}^{+} \tilde{\alpha}\right), & \operatorname{spt} Z_{0}^{+} \subset \pi^{-1}\left(C_{\left[0, \frac{1}{2}\right]}\right), \\
\partial Z_{1}^{-}=N^{*}\left(\tilde{\alpha}_{\frac{1}{2}}\right)-N^{*}\left(\phi_{1}^{-} \tilde{\alpha}\right), & \operatorname{spt} Z_{1}^{-} \subset \pi^{-1}\left(C_{\left[\frac{1}{2}, 1\right]}\right) .
\end{array}
$$

Thus for $W=Z_{1}^{+}-Z_{0}^{-},(3)$ follows from (1) and (2).

3.10 Proposition. Suppose $f: X \rightarrow Y$ and $g: Y \rightarrow Z$ are proper subanalytic maps. Then the pushforward operations under these maps satisfy the relation

$$
(g \circ f)_{*}=g_{*} \circ f_{*} .
$$

If the constructible function $\alpha$ has compact support in $X$ then

$$
\chi\left(f_{*} \alpha\right)=\chi(\alpha) .
$$

Proof. To prove (2), we imbed $X, Y$ in $\mathbb{R}^{m}, \mathbb{R}^{n}$ respectively, and then apply (3.9)(3) and the Gauss-Bonnet theorem (2.10). Using (2), we deduce (1) as follows. It suffices to prove that

$$
g_{*}\left(f_{*} \alpha\right)=(g \circ f)_{*} \alpha
$$


for $X$ compact and $\alpha=1_{X}$. Let $\beta=f_{*} \alpha$ and $\gamma=(g \circ f)_{*} \alpha$. We will show that $g_{*} \beta=\gamma$. Let $z \in Z$, let $X^{\prime}=(g \circ f)^{-1}(z), Y=g^{-1}(z)$, and let $f^{\prime}: X^{\prime} \rightarrow Y^{\prime}$ be the restriction of $f$. Then, since $f_{*}^{\prime}\left(1_{X^{\prime}}\right)=\beta \cdot 1_{Y^{\prime}}$,

$$
\begin{aligned}
\gamma(z) & =\chi\left(1_{X^{\prime}}\right) \\
& =\chi\left(f_{*}^{\prime}\left(1_{X^{\prime}}\right)\right) \quad \text { by }(2) \\
& =\chi\left(\beta \cdot 1_{Y^{\prime}}\right) \\
& =\left(g_{*} \beta\right)(z),
\end{aligned}
$$

as desired.

Remark. If $f: M \rightarrow N$ is a subanalytic map and $\beta$ is a constructible function on $N$, the pullback $f^{*} \beta$ is the constructible function on $M$ defined by

$$
\left(f^{*} \beta\right)(p)=\beta(f(p)) .
$$

It is easy to see that $(f \circ g)^{*}=g^{*} \circ f^{*}$, but $\chi\left(f^{*} \beta\right)$ is not equal to $\chi(\beta)$, in general.

3.11 Definition. Let $\alpha$ be a constructible function on the analytic manifold $M$. The dual of $\alpha$ is the constructible function $D_{M} \alpha$ on $M$ defined by

$$
\left(D_{M} \alpha\right)(p)=\chi\left(\alpha \cdot 1_{B_{\epsilon}^{\circ}(p)}\right)=\alpha(p)-\chi\left(\alpha \cdot 1_{S_{\epsilon}(p)}\right),
$$

where $B_{\epsilon}^{\circ}(p)$ is the open ball of radius $\epsilon>0$ about $p$ in an analytic metric on $M$, and $\epsilon$ is sufficiently small.

To see that $D_{M} \alpha$ does not depend on $\epsilon$ or on the choice of metric, note that if $\alpha=\sum_{i} n_{i} 1_{X_{i}}$ as in (3.2), then $D_{M} \alpha=\sum_{i} n_{i} D_{M} 1_{X_{i}}$. If $X$ is a closed subanalytic subset of $M$ and $p \in X$, then

$$
\left(D_{M} 1_{X}\right)(p)=\chi_{p}(X)
$$

where $\chi_{p}(X)$ is the local Euler characteristic of $X$ at $p$,

$$
\chi_{p}(X)=\sum_{i} \operatorname{rank} H_{i}(X, X \backslash\{p\}) .
$$

Furthermore, $\chi_{p}(X)=1-\chi(L)$, where $L$ is a subanalytic link of $p$ in $X(c f$. [CK]).

3.12 Theorem. If $\alpha$ is a constructible function on the oriented analytic n-manifold $M$, then

$$
(-1)^{n} a_{*} N_{M}^{*}(\alpha)=N_{M}^{*}\left(D_{M} \alpha\right)
$$

Proof. Embedding $M$ in a Euclidean space, we may assume by the product formula (2.7) that $M=\mathbb{R}^{n}$ and dim spt $\alpha \leq n-2$. Let $N^{*}=N_{\mathbb{R}^{n}}^{*}$ and $D=D_{\mathbb{R}^{n}}$. Using the isomorphism (3.6) between Legendrian cycles on $S^{*} \mathbb{R}^{n}$ and constructible functions on $\mathbb{R}^{n}$, there is a linear operator $A$ such that $(-1)^{n} a_{*} N^{*}(\alpha)=N^{*}(A \alpha)$; we must show that $A=D$. By the triangulation theorem for subanalytic sets it is enough to prove that $A\left(1_{\Delta}\right)=D\left(1_{\Delta}\right)$ for every subanalytic simplex $\Delta \subset \mathbb{R}^{n}$.

Let $k=\operatorname{dim} \Delta$, and let $f: B \rightarrow \Delta$ be a subanalytic homeomorphism from a $k$-ball $B$ to $\Delta$. Then

$$
\begin{gathered}
f_{*}\left(1_{B}\right)=1_{\Delta}, \quad f_{*}\left(1_{\partial B}\right)=1_{\partial \Delta}, \\
D\left(1_{B}\right)=(-1)^{k}\left(1_{B}-1_{\partial B}\right), \quad D\left(1_{\Delta}\right)=(-1)^{k}\left(1_{\Delta}-1_{\partial \Delta}\right),
\end{gathered}
$$


and therefore

$$
f_{*} D\left(1_{B}\right)=D\left(1_{\Delta}\right)
$$

Taking $n$ large enough we may assume that $B$ and $\Delta$ are embedded in $\mathbb{R}^{n}=$ $\mathbb{R}^{m} \times \mathbb{R} \times \mathbb{R}^{n-m-1}$ as the ends of the mapping cylinder $C$ of $f$. Consider the constructible function $\widetilde{1_{B}}=1_{C}-\left(1_{C_{0}}+1_{C_{1}}\right)$ on $C$. Then by (3.9)

$$
\begin{aligned}
& \phi_{0}^{+}\left(\widetilde{1_{B}}\right)=1_{B}, \\
& \phi_{1}^{-}\left(\widetilde{1_{B}}\right)=f_{*}\left(1_{B}\right)=1_{\Delta} .
\end{aligned}
$$

Now we claim that

$$
A\left(\widetilde{1_{B}}\right)_{t}=D\left(\widetilde{1_{B}}\right)_{t}, \quad 0<t<1
$$

If $0<s<t<1$, then the map $\psi_{s, t}: \mathbb{R}^{n} \rightarrow \mathbb{R}^{n}$,

$$
\psi_{s, t}(x, u, y)=\left(\frac{s}{t} x, s+t-u, \frac{1-s}{1-t} y\right),
$$

is an analytic isomorphism taking $\left(\widetilde{1_{B}}\right)_{t}$ to $\left(\widetilde{1_{B}}\right)_{s}$. Let $\Psi_{s, t}: S^{*} \mathbb{R}^{n} \rightarrow S^{*} \mathbb{R}^{n}$ be the isomorphism induced by $\psi_{s, t}$. Then

$$
\begin{aligned}
N^{*} A\left(\widetilde{1_{B}}\right)_{s} & =(-1)^{n} a_{*} N^{*}\left(\widetilde{1_{B}}\right)_{s} \\
& =(-1)^{n} a_{*}\left(\Psi_{s, t}^{-1}\right)_{*} N^{*}\left(\widetilde{1_{B}}\right)_{t} \\
& =\left(\Psi_{s, t}^{-1}\right)_{*}(-1)^{n} a_{*} N^{*}\left(\widetilde{1_{B}}\right)_{t} \\
& =\left(\Psi_{s, t}^{-1}\right)_{*} N^{*} A\left(\widetilde{1_{B}}\right)_{t} \\
& =N^{*}\left(\psi_{s, t}\right)_{*} A\left(\widetilde{1_{B}}\right)_{t},
\end{aligned}
$$

so by the isomorphism theorem (3.6), $A\left(\widetilde{1_{B}}\right)_{s}=\left(\psi_{s, t}\right)_{*} A\left(\widetilde{1_{B}}\right)_{t}$. Hence $A\left(\widetilde{1_{B}}\right)_{t}=\tilde{\beta}_{t}$ for some constructible function $\beta$ supported on $B$. The specialization theorem (3.7) yields

$$
\begin{aligned}
N^{*} A\left(1_{B}\right) & =(-1)^{n} a_{*} N^{*}\left(1_{B}\right) \\
& =(-1)^{n} a_{*} \lim _{t \downarrow 0} N^{*}\left(\widetilde{1_{B}}\right)_{t} \quad \text { by }(1) \\
& =\lim _{t \downarrow 0}(-1)^{n} a_{*} N^{*}\left(\widetilde{1_{B}}\right)_{t} \\
& =\lim _{t \downarrow 0} N^{*} A\left(\widetilde{1_{B}}\right)_{t} \\
& =\lim _{t \downarrow 0} N^{*} \tilde{\beta}_{t} \\
& =N^{*}(\beta),
\end{aligned}
$$

so $\beta=A\left(1_{B}\right)$ by (3.6). Thus $A\left(\widetilde{1_{B}}\right)_{t}=\left(A\left(1_{B}\right) \tilde{)}_{t}\right.$. On the other hand, $A\left(1_{B}\right)=$ $D\left(1_{B}\right)$ by a direct computation, and $\left(D\left(1_{B}\right)\right)_{t}=D\left(\widetilde{1_{B}}\right)_{t}$, which gives the claim (3). 
Therefore by (3.7) and (3.9),

$$
\begin{aligned}
N^{*} A\left(1_{\Delta}\right) & =N^{*} A f_{*}\left(1_{B}\right) \\
& =(-1)^{n} a_{*} N^{*} f_{*}\left(1_{B}\right) \\
& =(-1)^{n} a_{*} \lim _{t \uparrow 1} N^{*}\left(\widetilde{1_{B}}\right)_{t} \quad \text { by }(2) \\
& =\lim _{t \uparrow 1}(-1)^{n} a_{*} N^{*}\left(\widetilde{1_{B}}\right)_{t} \\
& =\lim _{t \uparrow 1} N^{*} A\left(\widetilde{1_{B}}\right)_{t} \\
& =\lim _{t \uparrow 1} N^{*} D\left(\widetilde{1_{B}}\right)_{t} \quad \text { by }(3) \\
& =N^{*} f_{*} D\left(1_{B}\right) \quad \text { by }(2) \\
& =N^{*} D\left(1_{\Delta}\right),
\end{aligned}
$$

from which we conclude by $(3.6)$ that $A\left(1_{\Delta}\right)=D\left(1_{\Delta}\right)$.

3.13 Corollary. Let $\alpha$ be a constructible function on the oriented analytic manifold M. Then

$$
D_{M}\left(D_{M} \alpha\right)=\alpha .
$$

If $\alpha$ has compact support, then

$$
\chi\left(D_{M} \alpha\right)=\chi(\alpha) .
$$

Proof. By embedding $M$ in a Euclidean space, we may assume that the support of $\alpha$ has codimension at least 2. Then (1) follows from (3.12) and (3.6), and (2) follows from (3.12) and (2.10).

Remark. Corollary (3.13) has an elementary combinatorial proof, which uses the triangulation theorem.

3.14 Proposition. Let $f: M \rightarrow N$ be a subanalytic map, and let $\alpha$ be a constructible function on $M$ such that $f \mid \operatorname{spt} \alpha$ is proper. Then

$$
D_{N}\left(f_{*} \alpha\right)=f_{*}\left(D_{M} \alpha\right) .
$$

Proof. As in the proof of (3.12), we may assume that $M$ and $N$ are embedded in $\mathbb{R}^{n}$ as the ends of the mapping cylinder of $f$. We will show that $D\left(f_{*} \alpha\right)=f_{*}(D \alpha)$, where $D=D_{\mathbb{R}^{n}}$. Let $N^{*}=N_{\mathbb{R}^{n}}^{*}$. Using the notation of (3.9), we have

$$
\begin{aligned}
N^{*} D\left(f_{*} \alpha\right) & =(-1)^{n} a_{*} N^{*}\left(f_{*} \alpha\right) \quad \text { by }(3.12) \\
& =(-1)^{n} a_{*} \lim _{t \uparrow 1} N^{*}\left(\tilde{\alpha}_{t}\right) \quad \text { by }(3.7) \text { and }(3.9) \\
& =\lim _{t \uparrow 1}(-1)^{n} a_{*} N^{*}\left(\tilde{\alpha}_{t}\right) \\
& =\lim _{t \uparrow 1} N^{*}\left(D \tilde{\alpha}_{t}\right) \quad \text { by }(3.12) \\
& =N^{*}\left(f_{*}(D \alpha)\right) \quad \text { by }(3.7) \text { and }(3.9),
\end{aligned}
$$

which implies $D\left(f_{*} \alpha\right)=f_{*}(D \alpha)$ by (3.6). 
3.15 Mod 2 constructible functions. Let $M$ be a real analytic manifold. The function $\alpha: M \rightarrow \mathbb{Z} / 2 \mathbb{Z}$ is (subanalytically) constructible if $\alpha^{-1}(0)$, and hence $\alpha^{-1}(1)$, are subanalytic sets. The properties of $\mathbb{Z} / 2 \mathbb{Z}$-valued constructible functions are parallel to the properties of $\mathbb{Z}$-valued constructible functions. The mod 2 Euler characteristic and the mod 2 conormal cycle are defined as above: If $\alpha=\sum_{i} n_{i} 1_{X_{i}}$ is a locally finite sum with $n_{i} \in \mathbb{Z} / 2 \mathbb{Z}$ and $X_{i}$ closed subanalytic, then

$$
\begin{gathered}
\chi(\alpha)=\sum_{i} n_{i} \chi\left(X_{i}\right), \\
\mathcal{N}_{M}^{*}(\alpha)=\sum_{i} n_{i} \mathcal{N}_{M}^{*}\left(X_{i}\right) .
\end{gathered}
$$

Here $\chi\left(X_{i}\right)$ denotes the mod 2 Euler characteristic of $X_{i}$, and $\mathcal{N}_{M}^{*}\left(X_{i}\right)$ is the $\bmod$ 2 conormal cycle (2.13). Pushforward and duality of mod 2 constructible functions are defined as for $\mathbb{Z}$-valued constructible functions, and all the results of this section are true for mod 2 constructible functions. In particular, the specialization theorem (3.7) and the mapping cylinder proposition (3.9) are true mod 2; their proofs use the local Gauss-Bonnet theorem. The $\mathbb{Z} / 2 \mathbb{Z}$ versions of (3.10)-(3.14) can be deduced from the $\mathbb{Z}$ versions by embedding in Euclidean space and reduction mod 2.

\section{Geometric Stiefel-Whitney Classes}

Sullivan [S] discovered a local topological property of real analytic varieties which implies that the combinatorial Stiefel chains (1.1) are cycles, and hence that the Stiefel-Whitney homology classes are defined. We show that Sullivan's local Euler characteristic condition for a subanalytic set $X$ is equivalent to the antipodal symmetry of the conormal cycle of $X$. This observation leads to a new definition of Stiefel-Whitney classes. In order to prove the Deligne-Grothendieck pushforward formula, we define the Stiefel-Whitney classes for certain constructible functions on an analytic manifold.

The following definition is due to Sullivan $[\mathrm{S}][\mathrm{A}]$.

4.1 Definition. A $(\bmod 2)$ Euler space is a triangulable topological space $X$ such that for all $x \in X$ the local Euler characteristic $\chi_{x}(X)=\sum_{i} \operatorname{rank} H_{i}(X, X \backslash\{x\})$ is odd.

If $\mathrm{lk}_{x}(K)$ is the link of $x$ in a triangulation $K$ of $X$ then a neighborhood of $x$ in $X$ is homeomorphic to the cone on $\mathrm{lk}_{x}(K)$, so $\chi_{x}(X)=1-\chi\left(\mathrm{k}_{x}(K)\right)$. Thus $X$ is an Euler space if and only if $\chi\left(\mathrm{lk}_{x}(K)\right)$ is even for every $x \in X$ and every triangulation $K$ of $X$. A subanalytic set $X$ is an Euler space if and only if, for every $x \in X$, the subanalytic link of $x$ in $X(c f$. [CK]) has even Euler characteristic.

We generalize Sullivan's definition as follows.

4.2 Definition. The mod 2 constructible function $\alpha$ on the analytic manifold $M$ is an Euler function if

$$
D_{M} \alpha=\alpha .
$$

By the definition of the duality operator $D_{M}$, the subanalytic subset $X$ of the analytic manifold $M$ is an Euler space if and only if the characteristic function $1_{X}$ is an Euler function.

The following result is an immediate corollary of Theorem 3.12. Recall that $a: S^{*} M \rightarrow S^{*} M$ is the antipodal map of the cotangent ray space of $M$, and $\mathcal{N}_{M}^{*}(\alpha)$ is the mod 2 conormal cycle of $\alpha$. 
4.3 Theorem. The mod 2 constructible function $\alpha$ on $M$ is an Euler function if and only if

$$
a_{*} \mathcal{N}_{M}^{*}(\alpha)=\mathcal{N}_{M}^{*}(\alpha) .
$$

We will say that the mod 2 integral current $\mathcal{C}$ in the cotangent ray space $S^{*} M$ is symmetric if $a_{*} \mathcal{C}=\mathcal{C}$. Thus $\alpha$ is Euler if and only if $\mathcal{N}_{M}^{*}(\alpha)$ is symmetric, and the subanalytic set $X$ is an Euler space if and only if the conormal cycle of $X$ is symmetric.

Now we prove the following key result of Sullivan [S].

4.4 Theorem. Every real analytic set is an Euler space.

Proof. Let $X$ be a real analytic set, which we may assume to be an analytic variety in $\mathbb{R}^{n}$. Let $Z \subset \mathbb{C}^{n}$ be a complexification of $X$. By [F5] the conormal cycle of $Z$ in $\mathbb{C}^{n}$ is invariant under multiplication by $\lambda$ for all complex numbers $\lambda$ of modulus 1 ; in particular $a_{*} N_{\mathbb{C}^{n}}^{*}(Z)=N_{\mathbb{C}^{n}}^{*}(Z)$. Therefore by (4.3) $Z$ is an Euler space. Now if $x \in X$ and $\Sigma$ is a small sphere about $x$ in $\mathbb{C}^{n}$, then the complex conjugation map $\tau: \mathbb{C}^{n} \rightarrow \mathbb{C}^{n}$ is an automorphism of the link $Z \cap \Sigma$ with fixed-point set $X \cap \Sigma$. Therefore $\chi(Z \cap \Sigma) \equiv \chi(X \cap \Sigma)(\bmod 2)$. Thus $\chi(X \cap \Sigma)$ is even.

The Stiefel-Whitney homology classes for a compactly supported subanalytic Euler function are constructed using the projectivized conormal cycle. Let $\alpha$ be a mod 2 constructible function on the analytic $n$-manifold $M$. Let $\pi: \mathbb{P} T^{*} M \rightarrow M$ be the projectivized cotangent bundle of $M$. The conormal cycle $\mathcal{N}_{M}^{*}(\alpha)$ is a closed $(n-1)$-dimensional Legendrian integral current in the cotangent ray space $S^{*} M$. Let $q: S^{*} M \rightarrow \mathbb{P} T^{*} M$ be the quotient map.

4.5 Definition. The projectivized conormal cycle of $\alpha$ is the mod 2 current

$$
\mathbb{P} N_{M}^{*}(\alpha)=q_{*}\left(\mathcal{N}_{M}^{*}(\alpha)\llcorner U),\right.
$$

where $U$ is a subanalytic subset of $S^{*}(M)$ with $U \cap a U=\emptyset$ and $U \cup a U=S^{*} M$.

By (4.3), for $U$ as above, the conormal cycle satisfies

$$
a_{*}\left(\mathcal{N}_{M}^{*}(\alpha)\llcorner U)=a_{*} \mathcal{N}_{M}^{*}(\alpha)\left\llcorner a U=\mathcal{N}_{M}^{*}(\alpha)\llcorner a U .\right.\right.
$$

Therefore,

$$
\begin{aligned}
a_{*} \partial\left(\mathcal{N}_{M}^{*}(\alpha)\llcorner U)\right. & =\partial a_{*}\left(\mathcal{N}_{M}^{*}(\alpha)\llcorner U)\right. \\
& =\partial\left(\mathcal{N}_{M}^{*}(\alpha)\llcorner a U)\right. \\
& =\partial\left(\mathcal{N}_{M}^{*}(\alpha)\llcorner U)\right.
\end{aligned}
$$

since $\partial\left(\mathcal{N}_{M}^{*}(\alpha)\left\llcorner U+\mathcal{N}_{M}^{*}(\alpha)\llcorner a U)=\partial \mathcal{N}_{M}^{*}(\alpha)=0\right.\right.$, so

$$
\partial \mathbb{P} N_{M}^{*}(\alpha)=q_{*} \partial\left(\mathcal{N}_{M}^{*}(\alpha)\llcorner U)=0 .\right.
$$

Furthermore, $\mathcal{N}_{M}^{*}(\alpha)$ may be reconstructed from $\mathbb{P} N_{M}^{*}(\alpha)$ as the fiber product

$$
\mathcal{N}_{M}^{*}(\alpha)=\mathbb{P} N_{M}^{*}(\alpha) \times_{\mathcal{B}}(\llbracket+1 \rrbracket+\llbracket-1 \rrbracket),
$$

where $\mathcal{B}$ is the bundle $q: S^{*} M \rightarrow \mathbb{P} T^{*} M$; this property is sufficient to determine $\mathbb{P} N_{M}^{*}(\alpha)$, and shows that it is well-defined, independent of the choice of the set $U$ above. Furthermore, if $\operatorname{spt} \alpha$ is contained in the subanalytic set $X$, then $\operatorname{spt} \mathbb{P} N_{M}^{*}(\alpha) \subset \pi^{-1}(X)$. Let $\left[\mathbb{P} N_{M}^{*}(\alpha)\right]$ be the $\bmod 2$ homology class of $\mathbb{P} N_{M}^{*}(\alpha)$ in $\pi^{-1}(X)$. Let $\pi_{X}: \pi^{-1}(X) \rightarrow X$ be the restriction of $\pi: \mathbb{P} T^{*} M \rightarrow M$. We abbreviate $\mathbb{P} N_{M}^{*}(\alpha)$ to $\mathbb{P} N^{*}(\alpha)$. 
Let $\zeta_{M} \in H^{1}\left(\mathbb{P} T^{*} M ; \mathbb{Z} / 2 \mathbb{Z}\right)$ be the $\bmod 2$ Euler class (first Stiefel-Whitney class) of the tautological line bundle over $\mathbb{P} T^{*} M$, and let $w^{i}(M) \in H^{i}(M ; \mathbb{Z} / 2 \mathbb{Z})$, $i=0, \ldots, n$, be the Stiefel-Whitney classes of the tangent bundle of $M$. Consider the cohomology classes

$$
\gamma_{M}^{k}=\sum_{i} \zeta_{M}^{k-i} \smile w^{i}(M)
$$

in $H^{k}\left(\mathbb{P} T^{*} M ; \mathbb{Z} / 2 \mathbb{Z}\right), k \geq 0$, where $\smile$ denotes cup product.

4.6 Definition. Let $\alpha$ be a subanalytic Euler function on the analytic manifold $M$, with compact support contained in the subanalytic set $X$. For each $i \geq 0$, the $i$ th Stiefel-Whitney class of $\alpha$ in $X$ is

$$
\left(w_{X}\right)_{i}(\alpha)=\left(\pi_{X}\right)_{*}\left(\left[\mathbb{P} N^{*}(\alpha)\right] \frown \gamma_{M}^{n-i-1}\right)
$$

in $H_{i}(X ; \mathbb{Z} / 2 \mathbb{Z})$, where $\frown$ denotes cap product.

We will abbreviate $\left(w_{X}\right)_{i}(\alpha)$ to $w_{i}(\alpha)$; the set $X$ will be clear from context. This definition is parallel to Fu's definition of the Chern-MacPherson homology classes of a complex analytic variety [F5, 2.4].

First we observe that the Stiefel-Whitney classes are additive.

4.7 Proposition. Let $\alpha$ and $\beta$ be constructible functions on $M$ with compact support contained in the subanalytic set $X$. Then, for all $i \geq 0$,

$$
w_{i}(\alpha+\beta)=w_{i}(\alpha)+w_{i}(\beta) .
$$

Proof. We have

$$
\begin{aligned}
w_{i}(\alpha+\beta) & =\left(\pi_{X}\right)_{*}\left(\left[\mathbb{P} N^{*}(\alpha+\beta)\right] \frown \gamma_{M}^{n-i-1}\right) \\
& =\left(\pi_{X}\right)_{*}\left(\left[\mathbb{P} N^{*}(\alpha)+\mathbb{P} N^{*}(\beta)\right] \frown \gamma_{M}^{n-i-1}\right) \\
& =\left(\pi_{X}\right)_{*}\left(\left[\mathbb{P} N^{*}(\alpha)\right] \frown \gamma_{M}^{n-i-1}\right)+\left(\pi_{X}\right)_{*}\left(\left[\mathbb{P} N^{*}(\beta)\right] \frown \gamma_{M}^{n-i-1}\right) \\
& =w_{i}(\alpha)+w_{i}(\beta) .
\end{aligned}
$$

Next we check that our definition agrees with the classical definition for manifolds. If $E$ is a vector bundle of rank $r$ and $\zeta$ is the Euler class of the tautological line bundle on $\mathbb{P} E$, then we have the Wu-Hirsch relation [Hu, 16.2.6]

$$
\sum_{i} \zeta^{r-i} \smile w^{i}(E)=0
$$

If the base $B$ of $E$ is a compact manifold and $p: \mathbb{P} E \rightarrow B$ is the projection, (4.8) implies that for all $i \geq 0$

$$
p_{*} \zeta^{r+i-1}=\bar{w}^{i}(E),
$$

where $p_{*}$ is the Gysin homomorphism (fiber integration), $\bar{w}^{0}=1$ and

$$
\sum_{i} \bar{w}^{k-i}(E) \smile w^{i}(E)=0
$$

for all $k>0$.

In the following we let $w \cdot(\alpha)=w_{0}(\alpha)+w_{1}(\alpha)+\cdots \in H_{*}(X ; \mathbb{Z} / 2 \mathbb{Z})$ and $w^{\cdot}(E)=$ $w^{0}(E)+w^{1}(E)+\cdots \in H^{*}(B ; \mathbb{Z} / 2 \mathbb{Z})$ denote the total Stiefel-Whitney classes. 
4.10 Proposition. If the subanalytic set $X$ in the analytic manifold $M$ is a compact $C^{1}$ submanifold of dimension d, then $w_{i}\left(1_{X}\right)$ is the Poincaré dual of the StiefelWhitney cohomology class $w^{d-i}(X), i=0, \ldots, d$.

Proof. Since $X$ is a $C^{1}$ submanifold of $M$, the projectivized conormal cycle of $X$ is the mod 2 integral current given by the projectivized conormal bundle $\mathbb{P}\left(n^{*} X\right)$. Now

$$
\begin{aligned}
w \cdot\left(1_{X}\right) & =\left(\pi_{X}\right)_{*}\left(\left[\mathbb{P} N^{*}\left(1_{X}\right)\right] \frown\left(\sum \zeta_{M}^{i} \smile w^{*}(M)\right)\right) \\
& =\left(\pi_{X}\right)_{*}\left(\left[\mathbb{P} N^{*}\left(1_{X}\right)\right] \frown \sum \zeta_{M}^{i}\right) \frown w^{*}(M) \\
& =\left([X] \frown \bar{w}^{\cdot}\left(n^{*} X\right)\right) \frown w^{\cdot}(M) \quad \text { by }(4.9) \\
& =[X] \frown\left(\bar{w}^{\cdot}\left(n^{*} X\right) \smile w^{\cdot}(M)\right) \\
& =[X] \frown\left(\bar{w}^{\cdot}\left(n^{*} X\right) \smile\left(w^{\cdot}\left(n^{*} X\right) \smile w^{\cdot}(X)\right)\right) \\
& =[X] \frown w^{\cdot}(X),
\end{aligned}
$$

as claimed.

Next we prove a special case of the fact that the Stiefel-Whitney classes of the Euler function $\alpha$ on $X$ are independent of the embedding of $X$.

4.11 Lemma. Let $M, M^{\prime}$ be analytic manifolds, let $\varphi: M \rightarrow M^{\prime}$ be an analytic embedding, and let $\alpha$ be a compactly supported Euler function on $M$. Then $\varphi_{*} w \cdot(\alpha)=w \cdot\left(\varphi_{*} \alpha\right)$.

Proof. Let $n=\operatorname{dim} M, n^{\prime}=\operatorname{dim} M^{\prime}$. Identifying $M$ with $\varphi(M)$, the product formula (2.9) implies that the projectivized conormal cycle $\mathbb{P} N_{M^{\prime}}^{*}(\alpha)$ may be expressed as the join of $\mathbb{P} N_{M}^{*}(\alpha)$ with the projectivized normal bundle of $M$. More precisely, let $\widetilde{P}$ denote the blowup of $\left.\mathbb{P} T^{*} M^{\prime}\right|_{M}$ over $\mathbb{P} T^{*} M \cup \mathbb{P}\left(n^{*} M\right)$; i.e.,

$$
\begin{aligned}
\widetilde{P} & =\left\{\left([\xi, \eta],\left[\xi^{\prime}\right],\left[\eta^{\prime}\right]\right) \mid \xi \wedge \xi^{\prime}=0=\eta \wedge \eta^{\prime}\right\} \\
& \subset\left(\left.\mathbb{P} T^{*} M^{\prime}\right|_{M}\right) \times_{M} \mathbb{P} T^{*} M \times_{M} \mathbb{P}\left(n^{*} M\right),
\end{aligned}
$$

where the blowdown map $\sigma:\left.\widetilde{P} \rightarrow \mathbb{P} T^{*} M^{\prime}\right|_{M}$ is the projection onto the first factor. Then (2.9) implies that the blowup cycle $\widetilde{N}=\left(\sigma^{-1}\right)_{*}\left(\mathbb{P} N_{M^{\prime}}^{*}(X)\right)$, a closed mod 2 integral current on $\widetilde{P}$, may be expressed as the iterated fiber product

$$
\widetilde{N}=\left(\mathbb{P} N_{M}^{*}(\alpha) \times_{\mathcal{B}} \llbracket \mathbb{P}^{n^{\prime}-n-1} \rrbracket\right) \times_{\mathcal{S}} \llbracket \mathbb{P}^{1} \rrbracket,
$$

where $\mathcal{B}$ is the pullback to $\mathbb{P} T^{*} M$ of the $\mathbb{P}^{n^{\prime}-n-1}$ bundle $\mathbb{P}\left(n^{*} M\right) \rightarrow M$, and $\mathcal{S}$ is the $\mathbb{P}^{1}$ bundle over $\mathbb{P} T^{*} M \times_{M} \mathbb{P}\left(n^{*} M\right)$ given by the projection onto the second and third factors in (1).

Next observe from (1) that the pullback via $\sigma$ of the tautological line bundle over $\left.\mathbb{P} T^{*} M^{\prime}\right|_{M}$ is the tautological bundle over $\mathcal{S}$ :

$$
\sigma^{*} \mathcal{O}_{\left.\mathbb{P} T^{*} M^{\prime}\right|_{M}}(1)=\mathcal{O}_{\mathcal{S}}(1)
$$

On the other hand, as a bundle over $\mathbb{P} T^{*} M \times_{M} \mathbb{P}\left(n^{*} M\right)$,

$$
\mathcal{S}=\mathbb{P}\left(\mathcal{O}_{\mathbb{P} T^{*} M}(1) \oplus \mathcal{O}_{\mathbb{P}\left(n^{*} M\right)}(1)\right),
$$


so if we put $\zeta=w^{1}\left(\mathcal{O}_{\left.\mathbb{P} T^{*} M^{\prime}\right|_{M}}(1)\right), z=w^{1}\left(\mathcal{O}_{\mathbb{P} T^{*} M}(1)\right), \bar{z}=w^{1}\left(\mathcal{O}_{\mathbb{P}\left(n^{*} M\right)}(1)\right)$, then with (3) the fundamental relation (4.8) gives

$$
\begin{aligned}
0= & \left(\sigma^{*} \zeta\right)^{2}+\left(\sigma^{*} \zeta\right) \cdot w^{1}\left(\mathcal{O}_{\mathbb{P} T^{*} M}(1) \oplus \mathcal{O}_{\mathbb{P}\left(n^{*} M\right)}(1)\right) \\
& +w^{2}\left(\mathcal{O}_{\mathbb{P} T^{*} M}(1) \oplus \mathcal{O}_{\mathbb{P}\left(n^{*} M\right)}(1)\right) \\
= & \left(\sigma^{*} \zeta\right)^{2}+\left(\sigma^{*} \zeta\right)(z+\bar{z})+z \bar{z}
\end{aligned}
$$

by the Whitney sum formula. Multiplying (4) by successive powers of $\sigma^{*} \zeta$ and substituting for $\left(\sigma^{*} \zeta\right)^{2}$ via (4), we find inductively, for each positive integer $k$,

$$
\left(\sigma^{*} \zeta\right)^{k+1}+\left(\sigma^{*} \zeta\right)\left(\sum_{i+j=k} z^{i} \bar{z}^{j}\right)+z \bar{z}\left(\sum_{i+j=k-1} z^{i} \bar{z}^{j}\right)=0 .
$$

Let $w .(\alpha)$ be the Stiefel-Whitney class of $\alpha$ as a function on $M$, and let $w .^{\prime}(\alpha)$ be the Stiefel-Whitney class of $\alpha$ as a function on $M^{\prime}$. We have the following computation of $w .^{\prime}(\alpha)$. (For simplicity we use - to denote both cup and cap products.)

$$
\begin{aligned}
w^{\prime}(\alpha) & =\pi_{*}\left(\left[\mathbb{P} N_{M^{\prime}}^{*}(\alpha)\right] \cdot\left(\sum \zeta^{k}\right) \cdot w^{*}\left(\left.T^{*} M^{\prime}\right|_{M}\right)\right) \\
& =\pi_{*}\left([\tilde{N}] \cdot\left(\sum\left(\sigma^{*} \zeta\right)^{k}\right) \cdot w^{\cdot}\left(\left.T^{*} M^{\prime}\right|_{M}\right)\right) \\
& =\pi_{*}\left([\tilde{N}] \cdot\left(\sigma^{*} \zeta+z \bar{z}\right) \cdot\left(\sum z^{i}\right) \cdot\left(\sum \bar{z}^{j}\right) \cdot w^{\cdot}\left(\left.T^{*} M^{\prime}\right|_{M}\right)\right) \quad \text { by }(5) \\
& =\pi_{*}\left(\left(\left[\mathbb{P} N_{M}^{*}(X) \times_{\mathcal{B}} \llbracket \mathbb{P}^{n^{\prime}-n-1} \rrbracket\right]\right) \cdot\left(\sum z^{i}\right) \cdot\left(\sum \bar{z}^{j}\right) \cdot w^{\cdot}\left(T^{*} M\right) \cdot w^{\cdot}\left(n^{*} M\right)\right)
\end{aligned}
$$

by (2), since $\sigma^{*} \zeta$ pairs nontrivially with the fibers of $\mathcal{S}$. Factoring the projection $\pi$ through the projection $\mu$ of $\mathcal{B}$,

$$
w^{\prime}(\alpha)=\pi_{*}\left(\left[\mathbb{P} N_{M}^{*}(\alpha)\right] \cdot\left(\sum z^{i}\right) \cdot w^{\cdot}\left(T^{*} M\right) \cdot \mu_{*}\left(\sum \bar{z}^{j}\right) \cdot w^{*}\left(n^{*} M\right)\right) .
$$

Now the identity (4.8) for the bundle $E=n^{*} M$ may be written

$$
\mu_{*}\left(\sum \bar{z}^{j}\right) \cdot w^{\cdot}\left(n^{*} M\right)=1,
$$

whence

$$
w^{\prime}(\alpha)=\pi_{*}\left(\left[\mathbb{P} N_{M}^{*}(X)\right] \cdot\left(\sum z^{i}\right) \cdot w^{\cdot}\left(T^{*} M\right)\right)=w \cdot(\alpha),
$$

as desired.

Now we prove the basic pushforward property for Stiefel-Whitney classes.

4.12 Theorem. Let $\alpha$ be a mod 2 constructible function on the analytic manifold $M$, such that the support of $\alpha$ is compact and contained in the subanalytic set $X$. Let $Y$ be a subanalytic subset of the analytic manifold $N$, and let $f: X \rightarrow Y$ be a subanalytic map. Then, for all $i \geq 0$,

$$
f_{*} w_{i}(\alpha)=w_{i}\left(f_{*} \alpha\right) .
$$

Proof. By (4.11) we may assume $M=\mathbb{R}^{m}$ and $N=\mathbb{R}^{m}$. Let $C$ be the mapping cylinder of $f$ (3.9). Let $\lambda: X \rightarrow C$ and $\mu: Y \rightarrow C$ be the inclusion maps

$$
\begin{aligned}
& \lambda(x)=(x, 0, f(x)), \\
& \mu(y)=(0,1, y) .
\end{aligned}
$$

Then $\mu$ is a homotopy equivalence, with homotopy inverse $\nu(x, t, y)=y$, and $\lambda$ is homotopic to $\mu \circ f$, by the homotopy $h_{t}(x)=((1-t) x, t, f(x))$. Therefore if we 
reduce the relation (3.9)(3) modulo 2, the definition of Stiefel-Whitney classes (4.6) gives

$$
\mu_{*} w \cdot\left(f_{*} \alpha\right)=\lambda_{*} w \cdot(\alpha)=\mu_{*} f_{*} w \cdot(\alpha)
$$

so $w \cdot\left(f_{*} \alpha\right)=f_{*} w \cdot(\alpha)$.

Remark. Our proof of the pushforward formula for Stiefel-Whitney classes is analogous to the original combinatorial proof sketched by Sullivan using the mapping cylinder [S, p. 167].

We prove a specialization formula for the Stiefel-Whitney classes.

4.13 Proposition. Let $\alpha$ be a mod 2 constructible function on the analytic manifold $M$ such that $X=\operatorname{spt} \alpha$ is compact and has positive codimension. Let $g: M \rightarrow$ $\mathbb{R}$ be a subanalytic function. For $t \in \mathbb{R}$, put $\alpha_{t}=\alpha \cdot 1_{X_{t}}$. Suppose that there exists $\epsilon>0$ such that $\alpha_{t}$ is Euler for all $t \in(0, \epsilon]$ and $\mathcal{N}^{*}(\alpha)\left\llcorner(g \circ \pi)^{-1}(0, \epsilon]\right.$ is symmetric. Then the specialization $\phi_{0} \alpha$ is Euler, and

$$
\lim _{t \downarrow 0} w \cdot\left(\alpha_{t}\right)=w \cdot\left(\phi_{0} \alpha\right)
$$

in the following sense. For every neighborhood $U$ of $X_{0}$, for $t>0$ sufficiently small, $X_{t} \subset U$ and

$$
j_{t *} w \cdot\left(\alpha_{t}\right)=j_{0 *} w \cdot\left(\phi_{0} \alpha\right),
$$

where $j_{s}: X_{s} \rightarrow U$ is the inclusion map.

Proof. Given $U$, choose $h$ so that $X_{[0, h]} \subset U$. Since $\mathcal{N}^{*}(\alpha)\left\llcorner(g \circ \pi)^{-1}(0, \epsilon]\right.$ is symmetric, the mod 2 current $Z_{h}$ of (3.7) is antipodally symmetric, and hence it admits a projectivization $\mathbb{P} Z_{h}$. Furthermore $\mathcal{N}^{*}\left(\phi_{0} \alpha\right)=\mathcal{N}^{*}\left(\alpha_{h}\right)-\partial Z_{h}$ is antipodally symmetric, since $\alpha_{h}$ is Euler. Let $\pi_{U}: \pi^{-1}(U) \rightarrow U$ be the restriction of $\pi: \mathbb{P} T^{*} M \rightarrow M$. Now

$$
\begin{aligned}
j_{h *} w \cdot\left(\alpha_{h}\right)-j_{0 *} w \cdot\left(\phi_{0} \alpha\right) & =\left(\pi_{U}\right)_{*}\left(\left(\left[\mathbb{P} N^{*}\left(\alpha_{h}\right)\right]-\left[\mathbb{P} N^{*}\left(\phi_{0} \alpha\right)\right]\right) \cdot \gamma^{*}\right) \\
& =\left(\pi_{U}\right)_{*}\left(\left[\partial \mathbb{P} Z_{h}\right] \cdot \gamma^{*}\right) \\
& =0
\end{aligned}
$$

as desired.

Remark. A specialization formula for bivariant Stiefel-Whitney classes of piecewiselinear spaces has been proved by Fulton and MacPherson [FM] (cf. [E]), but under the assumption that the parameter map $g$ is piecewise-linear. The specialization formula for Chern classes is due to Verdier [V]; other proofs of Verdier's formula have been given by Sabbah [Sa] and Fu [F2].

The parallel between our definition of Stiefel-Whitney classes and Fu's definition of Chern classes gives the following result.

4.14 Theorem. Let a be a complex analytically constructible $\mathbb{Z}$-valued function on the complex manifold $M$ such that a has compact support contained in the complex analytic variety $X$. Let $\alpha$ be the mod 2 equivalence class of $a$. Then, for all $i \geq 0$,

$$
\begin{aligned}
w_{2 i}(\alpha) & =r\left(c_{i}(a)\right), \\
w_{2 i+1}(\alpha) & =0,
\end{aligned}
$$

where $r: H_{2 i}(X ; \mathbb{Z}) \rightarrow H_{2 i}(X ; \mathbb{Z} / 2 \mathbb{Z})$ denotes reduction mod 2 . 
Proof. Let $n$ be the complex dimension of $M$. Denote the real and complex projectivizations of $T^{*} M$ by $\mathbb{R P} T^{*} M$ and $\mathbb{C P} T^{*} M$ respectively. Thus $\mathbb{R} \mathbb{P} T^{*} M$ is an $\mathbb{R} \mathbb{P}^{1}$-bundle $\mathcal{H}$ over $\mathbb{C P} T^{*} M$ with projection $\mu$. The pullback of the tautological line bundle $\mathcal{O}_{\mathbb{C}}(1)$ over $\mathbb{C P} T^{*} M$ may be written

$$
\mu^{*} \mathcal{O}_{\mathbb{C}}(1)=\mathcal{O}_{\mathbb{R}}(1) \oplus \sqrt{-1} \mathcal{O}_{\mathbb{R}}(1) \cong \mathcal{O}_{\mathbb{R}}(1) \oplus \mathcal{O}_{\mathbb{R}}(1)
$$

as a real vector bundle, so if we put $\zeta=c^{1}\left(\mathcal{O}_{\mathbb{C}}(1)\right), z=w^{1}\left(\mathcal{O}_{\mathbb{R}}(1)\right)$, then

$$
\mu^{*} \zeta \equiv w^{2}\left(\mathcal{O}_{\mathbb{R}}(1) \oplus \mathcal{O}_{\mathbb{R}}(1)\right) \equiv z^{2} \quad(\bmod 2)
$$

by the Whitney sum formula. Let $\mathbb{R} \mathbb{P} N^{*}(\alpha)$ denote the projectivized conormal cycle of $\alpha$; it is a $(2 n-1)$-dimensional mod 2 integral current in $\mathbb{R P} T^{*} M$. Let $\mathbb{C P} N^{*}(a)$ denote the complex projectivized conormal cycle of $a$ in the sense of [F5]; it is a $(2 n-2)$-dimensional integral current in $\mathbb{C P} T^{*} M$. These conormal cycles satisfy

$$
\mathbb{R} \mathbb{P} N^{*}(\alpha) \equiv \mathbb{C P} N^{*}(a) \times_{\mathcal{H}} \llbracket \mathbb{R} \mathbb{P}^{1} \rrbracket \quad(\bmod 2) .
$$

Therefore

$$
\begin{aligned}
w \cdot(\alpha) & =\pi_{*}\left(\left[\mathbb{R P} N^{*}(\alpha)\right] \cdot\left(\sum z^{i}\right) \cdot w^{\cdot}\left(T^{*} M\right)\right) \\
& \equiv \pi_{*}\left(\left[\mathbb{C P} N^{*}(a) \times_{\mathcal{H}} \llbracket \mathbb{R} \mathbb{P}^{1} \rrbracket\right] \cdot(1+z) \cdot \mu^{*}\left(\sum \zeta^{j}\right) \cdot c^{\cdot}\left(T^{*} M\right)\right) \quad(\bmod 2) .
\end{aligned}
$$

The fibers of $\mathcal{H}$ pair nontrivially with $z$, so if we factor the projection $\pi$ through $\mu$ then fiber integration gives

$$
\begin{aligned}
w .(\alpha) & \equiv \pi_{*}\left(\left[\mathbb{C P} N^{*}(a)\right] \cdot\left(\sum \zeta^{i}\right) \cdot c^{\cdot}\left(T^{*} M\right)\right) \quad(\bmod 2) \\
& =c .(a) .
\end{aligned}
$$

Finally we show that our definition (4.6) of Stiefel-Whitney classes satisfies the Deligne-Grothendieck axioms.

If $M$ is an analytic manifold, let $E(M)$ be the group of Euler functions on $M$. For $\alpha \in E(M)$, let $\operatorname{spt} \alpha$ denote the support of $\alpha$. If $X$ is a subanalytic subset of the analytic manifold $M$, let

$$
F(X)=\{\alpha \in E(M) \mid \operatorname{spt} \alpha \subset X, \text { spt } \alpha \text { compact }\} .
$$

If $Y$ is a subanalytic subset of the analytic manifold $N$, and $f: X \rightarrow Y$ is a subanalytic map, then the pushforward $f_{*} \alpha(3.8)$ is an Euler function by (3.14), and $f_{*} \alpha$ has compact support contained in $Y$. Thus $f_{*}: F(X) \rightarrow F(Y)$, and if $g: Y \rightarrow Z$, then $(g \circ f)_{*}=g_{*} \circ f_{*}$ by (3.10). Thus $F$ is a functor from subanalytic sets to abelian groups, which we call the Euler constructible function functor.

For each integer $i \geq 0$ let $H_{i}$ be the functor from subanalytic sets to abelian groups given by $H_{i}(X)=H_{i}(X ; \mathbb{Z} / 2 \mathbb{Z})$, the $i$ th $\bmod 2$ homology group of $X$. If $X$ is a compact $d$-dimensional analytic manifold, let $[X] \in H_{d}(X ; \mathbb{Z} / 2 \mathbb{Z})$ be the fundamental class of $X$, and for each $j \geq 0$ let $w^{j}(X) \in H^{j}(X ; \mathbb{Z} / 2 \mathbb{Z})$ be the $j$ th Stiefel-Whitney class of the tangent bundle of $X$. Now we state the fundamental existence and uniqueness theorem for Stiefel-Whitney homology classes. It is a generalization of Sullivan's theorem (1.1).

4.15 Theorem. For each $i \geq 0$ there exists a unique additive natural transformation $w_{i}$ from $F$ to $H_{i}$ such that if $X$ is a compact analytic d-manifold then $w_{i}\left(1_{X}\right)$ is Poincaré dual to $w^{d-i}(X)$. 
In other words, $w_{i}$ is a function which assigns to each Euler function $\alpha$ with compact support contained in $X$ an $i$-dimensional mod 2 homology class $w_{i}(\alpha)$ of $X$ such that

1. $w_{i}(\alpha+\beta)=w_{i}(\alpha)+w_{i}(\beta)$ for all $\alpha, \beta$,

2. $f_{*} w_{i}(\alpha)=w_{i}\left(f_{*} \alpha\right)$ for all $\alpha$ and all $f: X \rightarrow Y$,

3. $w_{i}\left(1_{X}\right)=[X] \frown w^{d-i}(X)$ for all compact analytic $d$-manifolds $X$.

These properties are analogous to the Deligne-Grothendieck axioms for Chern classes of complex analytic varieties $[\mathrm{M}]$. Fulton and MacPherson [FM, Thm. 6A, p. 64] (corrected by El Hauoari [E]) have proved a bivariant version of (4.15), but only in the piecewise-linear category.

Proof. To prove existence we define $w_{i}$ as in (4.6). Additivity (1) was proved in (4.7), pushforward (2) was proved in (4.12), and the manifold property (3) was proved in (4.10).

Uniqueness follows from the representability of mod 2 homology by analytic manifolds, as in the proof of Sullivan's theorem (1.1). Let $X \subset M$, and let $\alpha$ be an Euler function on $M$ such that spt $\alpha$ is compact and contained in $X$. Let $k$ be the dimension of $\operatorname{spt} \alpha$. Let $\mathcal{S}$ be a subanalytic stratification of $\operatorname{spt} \alpha$. Since $\alpha$ is Euler, each $(k-1)$-dimensional stratum of $\mathcal{S}$ is incident to an even number of $k$-dimensional strata. Thus the union of the $k$-dimensional strata of $\mathcal{S}$ is a mod 2 subanalytic cycle $Z$ (which represents $w_{k}(\alpha)$ ).

We claim that there exists a compact analytic $k$-manifold $V$ and a subanalytic map $f: V \rightarrow \operatorname{spt} Z$ of odd degree. By the Thom representability theorem [T, Thm. III.2], applied to the fundamental class $[Z] \in H_{k}(\operatorname{spt} Z ; \mathbb{Z} / 2 \mathbb{Z})$, there exists a compact $C^{\infty}$ manifold $V$ and a continuous map $f: V \rightarrow \operatorname{spt} Z$ such that $f_{*}[V]=$ $[Z]$. Since every compact $C^{\infty}$ manifold has an analytic structure $(c f$. [H, Thm. 4.7.1]), we can assume $V$ is analytic. Finally we choose subanalytic triangulations of $V$ and $\operatorname{spt} Z$, and then use simplicial approximation to replace $f$ by a simplicial (hence subanalytic) map homotopic to $f$.

Therefore if $\beta=\alpha-f_{*}\left(1_{V}\right)$ then $\beta$ is Euler and $\operatorname{dim}(\operatorname{spt} \beta)<k$. It follows by induction on $k$ that there exists a subanalytic map $\varphi: W \rightarrow X$ such that $W$ is a finite disjoint union of compact analytic manifolds and $\varphi_{*}\left(1_{W}\right)=\alpha$. Thus $w_{i}(\alpha)$ is uniquely determined by properties (1), (2) and (3).

\section{Polar CyCles}

Let $X$ be a compact subanalytic Euler space in $\mathbb{R}^{n}$, and let $A$ be a linear subspace of $\mathbb{R}^{n}$. We say that $A$ is general for $X$ if the following condition holds. Let $A^{*}$ be the subspace of $\mathbb{R}^{n *}$ corresponding to $A$ under the isomorphism $\mathbb{R}^{n} \cong \mathbb{R}^{n *}$ given by the Euclidean metric. Now $\mathbb{P} N^{*}(X) \subset \mathbb{P} T^{*}\left(\mathbb{R}^{n}\right) \cong \mathbb{R}^{n} \times \mathbb{P}\left(\mathbb{R}^{n *}\right)$, and $A$ is general for $X$ if there exists a subanalytic Whitney stratification $\mathcal{S}$ of $\operatorname{spt} \mathbb{P} N^{*}(X)$ such that $\mathbb{R}^{n} \times \mathbb{P}\left(A^{*}\right)$ is transverse to the strata of $\mathcal{S}$. For $k=1, \ldots, n$ the set of $k$-planes $A$ in $\mathbb{R}^{n}$ such that $A$ is general for $X$ is an open dense subset of the Grassmannian of $k$-planes in $\mathbb{R}^{n}$.

5.1 Definition. The polar cycle of the compact subanalytic Euler space $X$ with respect to a plane $A$ general for $X$, with $1 \leq \operatorname{dim} A \leq \operatorname{dim} X$, is the closed $\bmod 2$ current 


$$
\sigma(X, A)=\pi_{*}\left(\mathbb{P} N^{*}(X) \cdot\left(\mathbb{R}^{n} \times \mathbb{P}\left(A^{*}\right)\right)\right),
$$

where $\pi: \mathbb{P} T^{*} \mathbb{R}^{n} \rightarrow \mathbb{R}^{n}$ is the projection.

Here $\cdot$ denotes intersection of $\bmod 2$ currents. If $\operatorname{dim} A=i+1$, the homology class of the polar cycle $\sigma(X, A)$ is the Stiefel-Whitney class $w_{i}(X)$. The support of $\sigma(X, A)$ is contained in the set $\left\{x \in X \mid \exists \xi \in A^{*},(x, \xi) \in \operatorname{spt} N^{*}(X)\right\}$, the "singularity locus" of the orthogonal projection $X \rightarrow A$.

In fact $\sigma(X, A)$ is the generalization to subanalytic sets of the simplicial singularity cycle of Banchoff and McCrory [B] [Mc]. Let $X \subset \mathbb{R}^{n}$ be a simplicial Euler space; i.e., $X$ is an Euler space together with the structure of a linear simplicial complex $K$ in $\mathbb{R}^{n}: X=|K|$. Let $f: X \rightarrow \mathbb{R}^{i+1}$ be a map which is linear on each simplex of $K$. We say that $f$ is nondegenerate if, for every vertex $v$ of $K$, and for every set of distinct vertices $v_{0}, \ldots, v_{i+1}$ of the simplicial star of $v$, the affine span of the points $f\left(v_{0}\right), \ldots, f\left(v_{i+1}\right)$ is $\mathbb{R}^{i+1}$. The Euler singularity of $f$ is the mod 2 simplicial $i$-chain $\Sigma(f)$ of $K$ defined as follows (cf. [Mc, p. 373]). Let $S$ be an $i$-simplex of $K$, let $P$ be the affine hyperplane of $\mathbb{R}^{i+1}$ spanned by $f(S)$, and let $P^{+}, P^{-}$be the closures of the two components of $\mathbb{R}^{i+1} \backslash P$. Let $L$ be the simplicial link of $S$ in $K$, and let $L^{ \pm}=L \cap f^{-1}\left(P^{ \pm}\right)$. Then $\chi\left(L^{+}\right) \equiv \chi\left(L^{-}\right)(\bmod 2)$, and the coefficient of $S$ in the chain $\Sigma(f)$ is $1-\chi\left(L^{+}\right)(\bmod 2)$.

5.2 Proposition. Let $X$ be a simplicial Euler space in $\mathbb{R}^{n}$, let $A$ be a linear subspace of $\mathbb{R}^{n}$, and let $p: \mathbb{R}^{n} \rightarrow A$ be the orthogonal projection. If $p \mid X$ is nondegenerate then $A$ is general for $X$, and

$$
\sigma(X, A)=\Sigma(p \mid X)
$$

Proof. Let $N \subset T\left(\mathbb{R}^{n}\right)$ be the set of normal vectors of $X$; i.e., $(x, u) \in N$ if and only if $\left(x, \xi_{u}\right) \in \operatorname{spt} N^{*}(X)$, where $\xi_{u}(v)=\langle u /|u|, v\rangle$. The set $N$ is a conical subset of $T\left(\mathbb{R}^{n}\right)$; in other words, if $(x, u) \in N$ and $t>0$, then $(x, t u) \in N$. The plane $A$ is general for $X$ if and only if there is a conical subanalytic Whitney stratification $\mathcal{G}$ of $N$ such that $\mathbb{R}^{n} \times A$ is transverse to the strata of $\mathcal{G}$.

Let $X=|K|$. We will show that there is a canonical conical stratification $\mathcal{G}$ of $N$ associated to the triangulation $K$, and that $\mathbb{R}^{n} \times A$ is transverse to the strata of $\mathcal{G}$ if $p$ is nondegenerate.

If $S$ is a (closed) $k$-simplex of $K$, let $S^{o}$ be the interior of $S$ in the affine $k$ plane spanned by $S$, and let $S^{\perp}$ be the $(n-k)$-dimensional linear subspace of $\mathbb{R}^{n}$ orthogonal to $S$. Let $\mathcal{F}(S)$ be the conical stratification of $S^{o} \times S^{\perp}$ defined as follows. Let $\mathcal{T}$ be the set of all $(k+1)$-simplices of $K$ which have $S$ as a face. For each $T \in \mathcal{T}, T^{\perp}$ is a hyperplane of $S^{\perp}$. We let $\mathcal{E}(S)$ be the stratification of $S^{\perp}$ induced by this collection of hyperplanes. More precisely, if $v, w \in S^{\perp}$, let $[v, w]$ be the line segment between $v$ and $w$. Then $v$ and $w$ are in the same stratum of $\mathcal{E}(S)$ if for all $T \in \mathcal{T}$ either $[v, w] \subset T^{\perp}$ or $[v, w] \cap T^{\perp}=\emptyset$. Let $\mathcal{F}(S)=\left\{S^{o} \times E \mid E \in \mathcal{E}(S)\right\}$.

Let $N^{\prime}=\bigcup_{S}\left(S^{o} \times S^{\perp}\right)$, and consider the stratification $\mathcal{F}$ of $N^{\prime}$ given by $\mathcal{F}=$ $\bigcup_{S} \mathcal{F}(S)$. Now $N^{\prime} \subset N$, and it follows from the multiplicity formula (2.3) that $N$ is a union of strata of $\mathcal{F}$. We define the stratification $\mathcal{G}$ of $N$ by $\mathcal{G}=\{F \in \mathcal{F} \mid F \subset N\}$.

Now $p \mid X: X \rightarrow A$ is nondegenerate if and only if for all $S \in K$, the rank of $p$ restricted to the affine span of $S$ is $\min \{\operatorname{dim} S, \operatorname{dim} A\}$. This is equivalent to the statement that $A$ is transverse to $S^{\perp}$. Therefore if $p \mid X$ is nondegenerate then $\mathbb{R}^{n} \times A$ is transverse to the strata of $\mathcal{F}$, and so $\mathbb{R}^{n} \times A$ is a fortiori transverse to the strata of $\mathcal{G}$. 
It follows from the definition of the polar cycle $\sigma(X, A)$ and the multiplicity formula (2.3) that the multiplicity of the cycle $\sigma(X, A)$ is constant on the interior of each $i$-simplex $S$ of $K$. We proceed to give a formula for this multiplicity $\iota_{S}$.

If $T, U$ are simplices of $K$, we write $T<U$ if $T$ is a proper face of $U$. Given $S \in K$, the following subcomplexes of $K$ are associated to $S$ : the boundary of $S$, $\partial S=\{T \mid T<S\}$; the star of $S, \operatorname{St}(S)=\{T \mid \exists U, T \leq U \geq S\}$; the boundary of the star of $S, \partial \operatorname{St}(S)=\{T \in \operatorname{St}(S) \mid T \nsupseteq S\}$; the link of $S, \operatorname{Lk}(S)=\{T \in$ $\partial \operatorname{St}(S) \mid \nexists U, T \geq U \leq S\}$. For any subcomplex $L$ of $K$, we let $|L|$ denote the union of the simplices of $L$.

Let $S$ be an $i$-simplex of $K$. For notational simplicity assume that the origin 0 is in the interior of $S$. Choose $\epsilon>0$ so that $\epsilon<d(0,|\partial \operatorname{St}(S)|)$. Let $B_{\epsilon}$ be the sphere of radius $\epsilon$ in $\mathbb{R}^{n}$ centered at 0 . Let $u$ be a unit vector in the line $S^{\perp} \cap A$, and let $\xi(v)=\langle u, v\rangle$. Choose $\delta>0$ so that $\delta<\epsilon$ and $\delta<|\xi(y)|$ for all $y$ such that $y \in T \cap S^{\perp} \cap \partial B_{\epsilon}$ for some $(i+1)$-simplex $T$ with $S<T$. Then, by (2.2) and the product formula (2.9),

$$
\begin{aligned}
\iota_{S} & =\left.\chi\left(X \cap S^{\perp} \cap B_{\epsilon} \cap \xi^{-1}[-h, \infty)\right)\right|_{h=-\delta} ^{h=+\delta} \\
& =\chi(C)-\chi\left(\ell^{+}\right),
\end{aligned}
$$

where

$$
\begin{gathered}
C=X \cap S^{\perp} \cap B_{\epsilon} \cap \xi^{-1}[-\delta, \delta], \\
\ell^{+}=X \cap S^{\perp} \cap B_{\epsilon} \cap \xi^{-1}(\delta) .
\end{gathered}
$$

Now $S^{\perp} \cap|\operatorname{St}(S)|$ is the cone from 0 over the set $S^{\perp} \cap|\partial \operatorname{St}(S)|$, which is homeomorphic to $L=|\operatorname{Lk}(S)|$. Thus $C$ is a cone, since it is the intersection of the convex neighborhood $S^{\perp} \cap B_{\epsilon} \cap \xi^{-1}[-\delta, \delta)$ of 0 and the cone $S^{\perp} \cap|\operatorname{St}(S)|$. So $\chi(C)=1$, and

$$
\iota_{S}=1-\chi\left(\ell^{+}\right)
$$

By radial projection $\ell^{+}$is homeomorphic to $X \cap S^{\perp} \cap \partial B_{\epsilon} \cap \xi^{-1}[\delta, \infty)$. By the choice of $\delta$,

$$
X \cap S^{\perp} \cap \partial B_{\epsilon} \cap \xi^{-1}[0, \delta] \cong\left(X \cap S^{\perp} \cap \partial B_{\epsilon} \cap \xi^{-1}(\delta)\right) \times[0, \delta] .
$$

Therefore $\ell^{+} \cong X \cap S^{\perp} \cap \partial B_{\epsilon} \cap \xi^{-1}[0, \infty)$, which is homeomorphic by radial projection to $S^{\perp} \cap|\partial \operatorname{St}(S)| \cap \xi^{-1}[0, \infty) \cong|\operatorname{Lk}(S)| \cap \xi^{-1}[0, \infty)=L^{+}$. Therefore

$$
\iota_{S}=1-\chi\left(L^{+}\right),
$$

which is the multiplicity of $\Sigma(p \mid X)$ along $S$. Thus $\sigma(X, A)=\Sigma(p \mid X)$.

Remark. The Banchoff-McCrory description of the Euler singularity cycle of a nondegenerate simplexwise-linear map can be generalized to stratified maps, and the preceding proposition can be generalized to this context using stratified Morse theory $[\mathrm{GM}]$.

Note that if $X \subset \mathbb{R}^{n}$ and $f: X \rightarrow \mathbb{R}^{i+1}$ is a nondegenerate simplexwise-linear map, then $\Sigma(f)=\Sigma(p \mid \Gamma)$, where $p: \mathbb{R}^{n} \times \mathbb{R}^{i+1} \rightarrow \mathbb{R}^{i+1}$ is the projection and $\Gamma$ is the graph of $f$. 
Following Banchoff and McCrory, if $X=|K|$ we define a map $f_{i}: X \rightarrow \mathbb{R}^{i+1}$, nondegenerate and linear on the simplices of the barycentric subdivision $K^{\prime}$, by setting

$$
f_{i}\left(b \Delta^{k}\right)=\left(k, k^{2}, \ldots, k^{i+1}\right),
$$

where $b \Delta^{k}$ is the barycenter of the $k$-simplex $\Delta^{k}$. Recall that the $i$ th Stiefel chain $s_{i}(K)$ is the sum of all the $i$-simplices in the barycentric subdivision of $K$.

5.3 Proposition. $\Sigma\left(f_{i}\right)=s_{i}(K)$.

Proof. The proof given for manifolds in [B, p. 345] goes through for Euler spaces with only one change: The link of a simplex is not necessarily a sphere, but it has even Euler characteristic.

This result was used by Banchoff and McCrory to prove that if $X$ is a simplicial Euler space, then the Euler singularity cycle $\Sigma(f)$ of a nondegenerate simplexwiselinear map $f: X \rightarrow \mathbb{R}^{i+1}$ represents the Stiefel-Whitney homology class $w_{i}(X)$. Prior to their work the only definition of Stiefel-Whitney homology classes for singular spaces was the combinatorial formula. Our geometric construction of StiefelWhitney homology classes and verification of the axioms, together with (5.2) and (5.3), gives a proof of the combinatorial formula for these classes. This proof is new even for manifolds.

Remark. The present approach may also be applied to the smooth case, i.e. to embedded curvilinear $\left(C^{1}\right)$ simplicial complexes. Just as in section 4 , such a complex $X$ in a manifold $M$ is an Euler space (dropping the subanalyticity condition) if and only if its conormal cycle $\mathcal{N}_{M}^{*}(X)$ is antipodally symmetric. Therefore the StiefelWhitney classes $w_{i}(X)$ may again be constructed by the formula (4.6). That these classes do not depend on the (piecewise smooth) embedding of $X$ follows from the arguments of (3.7) (specialization), (4.11) (independence of analytic embedding), and (4.12) (pushforward). The key points which require clarification here are the mass bounds on the current $Z$ in the specialization formula for the mapping cylinder of a piecewise smooth homeomorphism, and the existence of the blowup $\widetilde{N}$ in the proof of (4.11). Both of these results follow from the hypothesis that the maps in question are diffeomorphisms piecewise, and therefore all slicing procedures are uniformly transverse to some compatible triangulation. In particular, the expression for the Stiefel-Whitney classes in terms of polar cycles yields the classical combinatorial formula for the Stiefel-Whitney classes of a $C^{1}$ triangulated manifold $[\mathrm{HT}]$.

\section{REFERENCES}

[A] E. Akin, Stiefel-Whitney homology classes and bordism, Trans. A.M.S. 205 (1975), 341359. MR 50:11288

[B] T. Banchoff, Stiefel-Whitney homology classes and singularities of projections for polyhedral manifolds, Proc. Symp. Pure Math., vol. 27, part 1, A.M.S., 1975, pp. 333-347. MR 51:14090

[Be] K. Bekka, Regular stratifications of subanalytic sets, Bull. London Math. Soc. 25 (1993), 7-16. MR 93f:32013

[BH] A. Borel and A. Haefliger, La classe d'homologie fondamentale d'un espace analytique, Bull. Soc. Math. France 89 (1961), 461-513. MR 26:6990

[BV] D. Burghelea and A. Verona, Local homological properties of analytic sets, Manus. Math. 7 (1972), 55-62. MR 46:9386 
[BDK] J. L. Brylinski, A. Dubson and M. Kashiwara, Formule de l'indice pour les modules holonomes et obstruction d'Euler locale, C. R. Acad. Sci. Paris, ser. A, 293 (1981), 573576. MR 83a:32010

[C] S. Cairns, A simple triangulation method for smooth manifolds, Bull. A.M.S. 67 (1961), 389-390. MR 26:6978

[Ch] J. Cheeger, A combinatorial formula for Stiefel-Whitney classes, Topology of Manifolds (J. Cantrell and C. H. Edwards, eds.), Markham, New York, 1970, pp. 470-471. MR 42:3793

[CK] M. Coste and K. Kurdyka, On the link of a stratum in a real algebraic set, Topology 31 (1992), 323-336. MR 93d:14088

[E] M. ElHaouari, Sur les classes de Stiefel-Whitney en theorie bivariante, Bull. Soc. Math. Belgique (1987), 151-186. MR 88a:57033

[F1] J. Fu, Monge-Ampère functions I, Indiana U. Math. J. 38 (1989), 745-771. MR 91d:49048

[F2] - On Verdier's specialization formula for Chern classes, Math. Ann. 291 (1991), 247-251. MR 92k:32073

[F3] - Curvature of singular spaces via the normal cycle, Proc. Symp. Pure Math., vol. 54, part 2, A.M.S., 1993, pp. 211-221. MR 94f:53126

[F4] - Curvature measures of subanalytic sets, Amer. J. Math. 116 (1994), 819-880. MR 95g:32016

[F5] Curvature measures and Chern classes of singular varieties, J. Diff. Geometry 39 (1994), 251-280. MR 95e:32013

[FM] W. Fulton and R. MacPherson, Categorical framework for the study of singular spaces, Memoirs A.M.S. 31 (1981). MR 83a:55015

[GM] M. Goresky and R. MacPherson, Stratified Morse Theory, Springer-Verlag, New York, 1988. MR 90d:57039

[HT] S. Halperin and D. Toledo, Stiefel-Whitney homology classes, Annals of Math. 96 (1972), 511-525. MR 47:1072

[Ha1] R. Hardt, Slicing and intersection theory for chains modulo $\nu$ associated with real analytic varieties, Trans. A.M.S. 183 (1973), 327-340. MR 49:3195

[Ha2] _ Sullivan's local Euler characteristic theorem, Manus. Math. 12 (1974), 87-92. MR 49:3196

[Ha3] , Topological properties of subanalytic sets, Trans. A.M.S. 211 (1975), 57-70. MR 52:751

[Ha4] Stratification of real analytic mappings and images, Invent. Math. 28 (1975), 193-208. MR 51:8453

[Ha5] , Triangulation of subanalytic sets and proper light subanalytic maps, Invent. Math. 38 (1977), 207-217. MR 56:12302

[Ha6] , Semi-algebraic local triviality in semi-algebraic mappings, Amer. J. Math. 102 (1980), 291-302. MR 81d:32012

[HM] R. Hardt and C. McCrory, Steenrod operations in subanalytic homology, Compositio Math. 39 (1979), 333-371. MR 82b:55019

[HZ] R. Harvey and J. Zweck, Stiefel-Whitney currents (to appear).

[Hi1] H. Hironaka, Subanalytic sets, Number Theory, Algebraic Geometry and Commutative Algebra, Kinokuniya, Tokyo, 1973, pp. 453-493. MR 51:13275

[Hi2] - Triangulations of algebraic sets, Proc. Symp. Pure Math., vol. 29, A.M.S., 1975, pp. 165-185. MR 51:10331

[H] M. Hirsch, Differential Topology, Springer-Verlag, New York, 1976. MR 56:6669

$[\mathrm{Hu}]$ D. Husemoller, Fibre Bundles, second edition, Springer-Verlag, New York, 1975. MR 51:6805

[KS] M. Kashiwara and P. Schapira, Sheaves on Manifolds, Springer-Verlag, Berlin, 1990. MR 92a: 58132

[M] R. MacPherson, Chern classes for singular algebraic varieties, Annals of Math. 100 (1974), 423-432. MR 50:13587

[Mc] C. McCrory, Euler singularities and homology operations, Proc. Symp. Pure Math., vol. 27, part 1, A.M.S., 1975, pp. 371-380. MR 51:14089

[Sa] C. Sabbah, Quelques remarques sur la géométrie des espaces conormaux, Astérisque 130 (1985), 161-192. MR 87f:32031 
[Sc] P. Schapira, Operations on constructible functions, J. Pure Appl. Algebra (1991), 83-93. MR 92h:32012

[SY] M. Shiota and M. Yokoi, Triangulations of subanalytic sets and locally subanalytic manifolds, Trans. A.M.S. 286 (1984), 727-750. MR 86m:32014

[St] E. Stiefel, Richtungsfelder und Fernparallelismus in Mannigfaltigkeiten, Comm. Math. Helvetici (1936), 3-51.

[S] D. Sullivan, Combinatorial invariants of analytic spaces, Proc. Liverpool Singularities Symposium, Lecture Notes in Math., vol. 192, Springer-Verlag, 1971, pp. 165-169. MR 43:4063

[T] R. Thom, Quelques propriétés globales des variétés différentiables, Comm. Math. Helvetici 28 (1954), 17-86. MR 15:890a

[V] J.-L. Verdier, Spécialisation des classes de Chern, Astérisque 82-83 (1981), 149-159. MR 83m:14015

[W] H. Whitney, On the theory of sphere bundles, Proc. Nat. Acad. Sci. U.S.A. 26 (1940), 148-153. MR 1:220b

Department of Mathematics, University of Georgia, Athens, Georgia 30602

E-mail address: fu@math.uga.edu

E-mail address: clint@math.uga.edu 